\title{
Cambio organizacional, institucional y tecnológico: una aproximación desde la teoría actor-red y el trabajo institucional *
}

\author{
Organizational, Institutional and Technological Change: an Approach from Actor Network Theory and \\ Institutional Work
}

Mudança organizacional, institucional e tecnológica: uma abordagem da teoria ator-rede e o trabalho institucional

Viviana Andrea Gutiérrez Rincón ${ }^{a}$

Pontificia Universidad Javeriana, Colombia

vgutierrez@javerianacali.edu.co

ORCID: http://orcid.org/0000-0002-4584-2370

José Javier Aguilar Zambrano

Universidad Nacional de Colombia, Colombia

ORCID: http://orcid.org/0000-0002-0947-2886

Javier Enrique Medina Vásquez

Universidad del Valle, Colombia
DOI: https://doi.org/10.11144/Javeriana.cao32-59.coit Redalyc: http://www.redalyc.org/articulo.oa? id=cuadernoss 20561244005

Fecha de recepción: 28 Noviembre 2018

Fecha de aprobación: 18 Octubre 2019

Fecha de publicación: 15 Diciembre 2019

\section{Resumen:}

La interrelación entre cambio organizacional, tecnológico e institucional ha sido ampliamente estudiada desde diferentes teorías. Sin embargo, poco se ha explorado sobre écómo con la introducción de una nueva tecnología se entrelazan las propiedades materiales de la tecnología con los arreglos sociales del contexto donde se implementan, generando cambios en las tecnologías, organizaciones e instituciones?, convirtiéndose en nuestra pregunta de investigación. El estudio parte de una revisión de teorías de cambio organizacional, institucional y tecnológico, identificando diferencias y complementariedades entre sus conceptos, generando así una propuesta teórica para analizar la interacción de actores no-humanos con las prácticas institucionalizadas de actores humanos en la implementación de tecnologías desde la Teoría Actor-Red y el Trabajo Institucional.

Códigos JEL: B52, L20, O32, O33

Palabras clave: teoría actor-red, trabajo institucional, cambio institucional, cambio tecnológico, cambio organizacional.

\section{Abstract:}

The interrelation between organizational, technological and institutional change has been widely studied from different theories. However, little has been explored on how with the introduction of a new technology the material properties of the technology are intertwined with the social arrangements of the context where they are implemented, generating changes in the technologies, organizations and institutions?, becoming our research question. The study starts from a review of theories of organizational, institutional and technological change, identifying differences and complementarities between its concepts, thus generating a theoretical proposal to analyze the interaction of non-human actors with the institutionalized practices of human actors in the implementation of technologies from Actor-Network Theory and Institutional Work.

JEL Codes: B52, L20, O32, O33

Keywords: Actor network theory, institutional work, institutional change, technological change, organizational change.

\section{Resumo:}

A inter-relação entre mudança organizacional, tecnológica e institucional tem sido amplamente estudada a partir de diferentes teorias. No entanto, pouco foi explorado sobre como, com a introdução de uma nova tecnologia, as propriedades materiais da tecnologia se entrelaçam com os arranjos sociais do contexto em que são implementadas, gerando mudanças em tecnologias, organizações e instituições? tornando-se nossa questão de investigação. O estudo parte de uma revisão das teorias de mudança organizacional, institucional e tecnológica, identificando diferenças e complementaridades entre seus conceitos, gerando uma proposta teórica para analisar a interação de atores não humanos com as práticas institucionalizadas de atores humanos na implementação de tecnologias. Teoria Ator-Rede e Trabalho Institucional.

Notas de autor 
Palavras-chave: Teoria ator-rede, trabalho institucional, mudança institucional, mudança tecnológica, mudança organizacional.

\section{INTRODUCCIÓN}

La interrelación entre organizaciones, tecnologías e instituciones se ha evidenciado desde diferentes teorías de cambio organizacional, institucional y tecnológico. Por un lado, la relación entre tecnología y organizaciones ha sido ampliamente estudiada desde diversas disciplinas que centran su atención en la relación determinante desde la tecnología hacia las organizaciones, o viceversa. Desde la economía, por ejemplo, autores como Thompson y Bates (1957 en Zammuto, Griffith, Majchrzak, Dougherty, \& Faraj, 2007), Blau et al. (1976 en Leonardi \& Barley, 2010), Freeman et al. (1982 en Williams \& Edge, 1996), Perez (1983 en Williams \& Edge, 1996), Huber (1990 en Orlikowski \& Scott, 2008), y Aiman-Smith y Green (2002 en 1990 en Orlikowski $\&$ Scott, 2008), se han enfocado en los aspectos físicos de la tecnología y sus efectos sobre las organizaciones resultando en un determinismo tecnológico (Barley, 1986; Orlikowski \& Scott, 2008; Orlikowski, 2010).

Desde la sociología, específicamente, desde el constructivismo social, autores como Tris y Bamforth (1951 en Orlikowski \& Scott, 2008), Emery (1959 en Orlikowski \& Scott, 2008), Barley (1986), Lucas (1975 en Leonardi \& Barley, 2010), Robey (1979 en Leonardi \& Barley, 2010), Prasad (1993 en Orlikowski \& Scott, 2008), y Boudreau y Robey (2005 en Orlikowski \& Scott, 2008) se han enfocado en la tecnología como producto social, centrándose en la agencia humana como principal impulsor de esta relación, es decir, que los humanos podemos dar forma a nuestro ambiente para alcanzar nuestros objetivos (Leonardi \& Barley, 2008), resultando en una forma de determinismo social (Barley, 1986; Orlikowski \& Scott, 2008; Orlikowski, 2010).

Desde otra vertiente de la sociología, con la intención de responder a las críticas que se presentan a los estudios de la sociología de la ciencia y al constructivismo social, los estudios sociales de la ciencia y la tecnología se centran en estudiar la contribución de los procesos sociales en el desarrollo de nuevas tecnologías, a partir de la inclusión de la materialidad de la tecnología, (Bijker, 1995; Latham \& Sassen, 2005; Pickering, 1995). En este mismo sentido, autores como Callon (1986), Knorr-Cetina (1997) y Latour (2008) han planteado la disolución de los límites analíticos entre tecnologías y humanos, a través de la Teoría de Actor-Red, definiendo la agencia como el relato de la acción, es decir, es una explicación de cómo se hace algo con incidencia, de alguna manera, en el estado de las cosas (Latour, 2008).

Por otra parte, la relación entre cambio institucional y cambio organizacional ha sido estudiada desde la Teoría Institucional, por autores como Oliver (1991), Greenwood, Suddaby y Hinings (2002), y Seo y Creed (2002). Otro enfoque de esta misma teoría, denominado Trabajo Institucional, busca entender el cambio institucional desde el rol de los actores humanos en crear, transformar y mantener las prácticas institucionalizadas (Lawrence \& Suddaby, 2006; Lawrence, Suddaby \& Leca, 2009; Lawrence, Suddaby \& Leca, 2011; Lawrence, Leca \& Zilber, 2013), por lo que autores como Dover y Lawrence (2010), Thornton, Ocasio y Lounsbury (2012), Jones y Massa (2013), Raviola y Norbäck (2013), y Monteiro y Nicolini (2014) han identificado la importancia asignada a la agencia humana, resaltando la necesidad de involucrar la tecnología como objeto material con relación a las instituciones.

La propuesta parte de una revisión de teorías de cambio organizacional (Van de Ven \& Poole, 1995), institucional (Barley 1986; Battilana \& D’Aunno 2009; Clemens \& Cook, 1999) y tecnológico (Leonardi \& Barley, 2008; Orlikowski \& Scott, 2008), que han estudiado la relación entre organizaciones, instituciones y tecnología. De esta manera se identifican diferencias y complementariedades entre los conceptos estudiados, y a partir de la Teoría Actor-red y el Trabajo Institucional, se vinculan y analizan estos conceptos ofreciendo herramientas conceptuales para estudiar la interrelación entre actores no-humanos y humanos (Callon, 1986; Knorr-Cetina, 1997; Latour, 2008, 2015) en la implementación de nuevas tecnologías y sus efectos sobre las prácticas institucionalizadas. 
El presente estudio espera contribuir a la comprensión de cómo con la introducción de una nueva tecnología se entrelazan las propiedades materiales de la tecnología con los arreglos sociales del contexto donde se implementan, generando cambios en las tecnologías, organizaciones e instituciones (Lawrence et al., 2013; Montero \& Nicolini, 2014; Raviola \& Norback, 2013.

Este documento se estructura en cuatro partes. Primero, se expone una revisión de las publicaciones científicas sobre cambio organizacional, cambio institucional y cambio tecnológico. Segundo, se identifica la relación que se ha presentado desde la literatura entre cambio organizacional, tecnológico e institucional. Tercero, se presenta la propuesta teórica que consta de tres proposiciones, complementando los conceptos revisados a partir de la Teoría de Actor-Red y Trabajo Institucional. Finalmente se presentan las conclusiones junto con las recomendaciones para futuras investigaciones.

\section{TEORÍAS DE CAMBIO ORGANIZACIONAL, INSTITUCIONAL Y TECNOLÓGICO}

El cambio organizacional, cambio institucional y cambio tecnológico se han constituido en temas de vigencia en las publicaciones científicas en management. En figura 1 se muestra el número de artículos que desde el año 2001 hasta junio del 2018 han tratado el tema en la Web of Science; se observa un crecimiento constante en el número de publicaciones en las tres temáticas, siendo el cambio organizacional el de mayor número de publicaciones con un total de 2259, mientras que en este mismo periodo el cambio tecnológico cuenta con 741 publicaciones y el cambio institucional con 486 publicaciones.

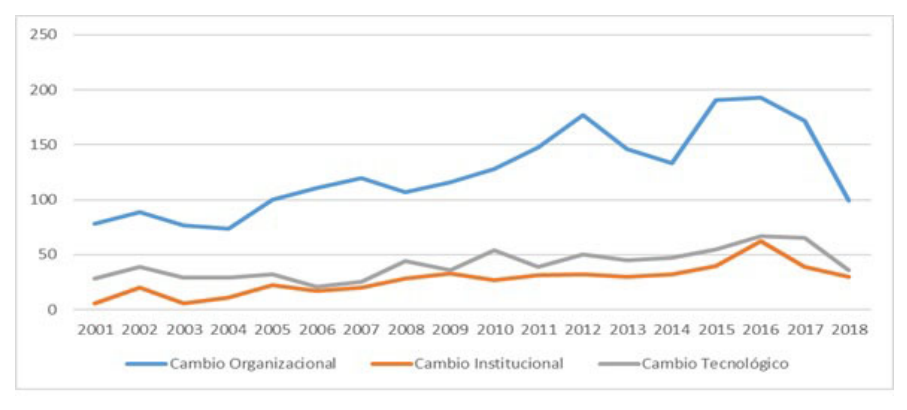

FIGURA 1

Publicación de artículos científicos en cambio organizacional, institucional y tecnológico Fuente: elaboración propia con datos Web of Science.

El dominio del cambio organizacional sobre el cambio institucional y tecnológico también se puede observar en las publicaciones más relevantes en el tema de management, según su factor de impacto (ver tabla $1)$.

TABLA 1

Revistas de impacto en el tema management

\begin{tabular}{lcccc}
\hline \multicolumn{1}{c}{ Nombre de la Revista } & $\begin{array}{c}\text { Organizational } \\
\text { change }\end{array}$ & $\begin{array}{c}\text { Institutional } \\
\text { change }\end{array}$ & $\begin{array}{c}\text { T echnological } \\
\text { change }\end{array}$ & Total \\
\hline Academy of Management Review & 25 & 12 & 8 & 45 \\
Jounal of Management & 28 & 4 & 7 & 39 \\
Academy of Management Jowond & 56 & 25 & 11 & 92 \\
Adbrinistrative Science Quarterly & 30 & 19 & 4 & 53 \\
Strategic Management Jownal & 43 & 10 & 36 & 89 \\
MIS Quarterly & 35 & 1 & 3 & 39 \\
Jounal of Management Studies & 43 & 18 & 8 & 69 \\
Research Policy & 17 & 19 & 112 & 148 \\
Organization Stucies & 68 & 49 & 3 & 120 \\
Organization Science & 85 & 39 & 30 & 154 \\
Total & 430 & 196 & 222 & \\
\hline
\end{tabular}

Fuente: elaboración propia con datos de Web of Science. 
Para comprender la importancia del cambio organizacional, institucional y tecnológico, en los estudios organizacionales, se hizo una revisión de algunas de las teorías que los abordan (Van de Ven \& Poole, 1995; Barley 1986; Battilana \& D’Aunno 2009; Clemens \& Cook, 1999; Leonardi \& Barley, 2008; Orlikowski \& Scott, 2008), cuya síntesis se presentan a continuación.

\section{Cambio Organizacional}

La explicación de cómo y por qué las organizaciones cambian ha sido una búsqueda central y constante de los estudios organizacionales, la cual ha generado una diversidad de teorías y conceptos que ofrecen visiones parciales y contrastantes del cambio organizacional. Van de Ven y Poole (1995) realizaron una revisión de diversas teorías de cambio organizacional, a partir de la cual propusieron una tipología de cuatro escuelas de pensamiento que denominaron: ciclo de vida, teleología, dialéctica y evolucionaria; sobre la base de estas tipologías se busca explicar el cómo y el porqué del cambio organizacional, centrándose en las condiciones del proceso y no en sus consecuencias y salidas.

Según los autores, estas tipologías se diferencian por dos tipos de características, por la unidad de análisis y por el modo de cambio. Por un lado, la unidad de análisis hace referencia a si el cambio se genera en una entidad singular, entendiéndose por entidad "un trabajo individual, un trabajo en grupo, una estrategia organizacional, un programa, un producto o la totalidad de una organización” (Van de Ven \& Poole, 1995, p. 512) o en múltiples entidades. Por otro lado, por el modo de cambio que se divide en prescriptivo y constructivista, donde el prescriptivo hace referencia a que la secuencia de eventos de cambio están determinados previamente y son predecibles, debido a que sigue el patrón de estados anteriores, generando cambios de primer-orden, es decir pequeños cambios que en el largo plazo pueden acumularse para producir un gran cambio; mientras que el proceso constructivista hace referencia a una progresión que es construida y emerge mientras el proceso de cambio se despliega, generando cambios de segundo-orden, es decir cambios que rompen con los supuestos y marcos básicos utilizados hasta entonces (Van de Ven \& Poole, 1995).

La teoría del ciclo de vida asume la metáfora del crecimiento orgánico para identificar las etapas de desarrollo de una entidad organizacional, desde el inicio hasta el final. Desde esta teoría el cambio es percibido como prescriptivo, en tanto lineal e irreversible, donde se sigue una secuencia única de etapas, que son acumulativas y conectadas entre sí, que pudieron generarse por un programa, regla, lógica o institución (Van de Ven \& Poole, 1995).

La teoría teleológica explica que la entidad existe por un propósito y se adapta para dar alcance a un objetivo o estado final deseado, para el cual toma acciones de forma individual o en interacción con otros. Es importante resaltar que desde esta teoría no se plantea una trayectoria específica para dar alcance a su objetivo, sino que a partir de sus aprendizajes puede modificar sus objetivos y cursos de acción, generando un cambio de manera constructivista (Van de Ven \& Poole, 1995).

La teoría dialéctica asume que la entidad existe en un mundo pluralista de eventos, fuerzas y valores en conflicto que compiten entre sí por la dominación y el control. Desde esta escuela la estabilidad y el cambio son constructivistas, ya que son explicadas por el balance entre el poder de las entidades que se oponen, que los autores explican como tesis (estado actual), antítesis (desafío o valores, fuerzas o eventos de oposición) y síntesis (nuevo estado que conjuga la tesis y antítesis); a pesar de esto los autores reconocen también procesos donde se mantiene la tesis actual así como procesos donde la oposición moviliza suficiente poder para imponer la antítesis (Van de Ven \& Poole, 1995).

Finalmente, la teoría evolucionaria asume que el cambio ocurre en las formas organizacionales y ocurre en múltiples entidades, a través de un ciclo continuo de variación, selección y retención, es decir, con un modo prescriptivo. Donde la selección de una forma organizacional ocurre por la competencia por recursos escasos, la retención se da cuando se mantiene o perpetúa una práctica establecida y la variación estimula la selección de una nueva forma organizacional (Van de Ven \& Poole, 1995). 
Autores como Meyer, Brooks y Goes (1990) y Scott, Ruef, Mendel y Caronna (2000) reiteran la importancia de los cambios de primer y segundo orden, los cuales relacionan con la magnitud y la escala de los cambios. Meyer et al. (1990) proponen un marco conceptual que diferencia el cambio organizacional según el nivel de análisis, es decir una organización o campo organizacional, y según los modos de cambio que clasifican en continuo versus discontinuo. El cambio continuo o de primer orden ocurre dentro de un sistema estable y en periodos de equilibrio, mientras que el cambio discontinuo o de segundo orden genera transformación de las propiedades fundamentales del sistema durante periodos de disrupción (Meyer et al., 1990; Nadler \& Tushman, 1995).

En este marco conceptual, Meyer et al. (1990) clasifican las teorías sobre la alineación de las organizaciones con sus ambientes en adaptación, evolución, metamorfosis y revolución. Las teorías clasificadas como adaptación son las que plantean que las organizaciones siguen sus ambientes de forma continua y ajustan de forma incremental sus productos, estructuras y procesos a estos ambientes y con los cuales pueden tener diferentes niveles de dependencia. Las teorías de evolución se enfocan también en los cambios continuos o incrementales, pero a nivel de una industria o campo organizacional, cambios que se pueden dar por selección natural, es decir, que la entrada o salida de empresas dentro de una industria puede generar que la población de empresas evolucione gradualmente para ajustarse a los cambios ambientales; o por las presiones que tienen las organizaciones a ajustarse a las expectativas normativas de sus ambientes institucionales con el objetivo de mantener o mejorar su legitimidad. Las teorías denominadas metamorfosis se enfocan en las rupturas de los marcos que existen dentro de la organización debido al progreso en las etapas de los ciclos de vida de la organización y avances tecnológicos, entre otros, generando cambios discontinuos. Finalmente, las teorías de revolución se enfocan en la restructuración y reconstitución de las industrias, de donde se plantea pueden surgir nuevas formas organizacionales.

Scott et al. (2000 argumentan que los procesos de cambio propuestos por Meyer et al. (1990 pueden ocurrir simultáneamente, y la incidencia o intensidad del cambio en un cuadrante puede influir en la incidencia o intensidad del cambio en otro, por lo que los cambios en los dos niveles, organización y campo organizacional, pueden estar conectados más no amarrados de manera determinística, ya que por ejemplo un periodo de cambio revolucionario en la industria puede ser asociado con una metamorfosis o transformación significativa de una organización.

Estos cambios denominados continuos o discontinuos, también han sido estudiado por autores como Rindova y Petkova (2007 quienes argumentan que, dependiendo del grado del cambio, enfocándose en el tecnológico, la innovación puede afectar la forma en que esta es percibida, dependiendo de su congruencia frente a los esquemas existentes, como son conocimiento, prácticas, significados y entendimientos compartidos. En este sentido, los cambios continuos o innovaciones incrementales son percibidos como congruentes debido a que se ajustan fácilmente con los esquemas existentes, mientras que los cambios discontinuos o innovaciones radicales pueden generar diversas incongruencias al introducir cambios significativos en los esquemas actuales y en las relaciones entre ellos (Nadler \& Tushman, 1995; Ridova \& Petkova, 2007.

Rindova y Petkova (2007 plantean que, para las innovaciones incrementales, los esquemas disponibles pueden ser utilizados directamente o requieren de pequeñas variaciones, que los usuarios podrían alcanzar mediante extensiones del esquema. Por otro lado, en la introducción de innovaciones radicales, los esquemas disponibles pueden tener una aplicabilidad limitada, por lo tanto, los usuarios solo llegan a entendimientos limitados y a una diversidad de interpretaciones de la nueva tecnología.

Christensen, Grossman y Hwang (2009 se refieren a las innovaciones incrementales como sostenibles porque buscan mantener la trayectoria existente del mercado, al mejorar el desempeño de las organizaciones que lo conforman, evaluado según las dimensiones o los atributos que son reconocidos por los usuarios, mientras que la innovación disruptiva no cumple con los parámetros de desempeño actuales del mercado. Christensen et al. (2009 relacionan este tipo de innovación a la tecnología disruptiva, definiendo el término 
disrupción como "una innovación que hace las cosas más simples y más asequibles" (Christensen et al., 2009, p. 1) con respecto a las tecnologías existentes en el mercado.

En la definición elaborada por Christensen et al. (2009) se utiliza el término innovación disruptiva, asemejándose a la definición de Rindova y Petkova (2007), que utiliza el término de innovación radical, en estas dos definiciones se plantea que estos tipos de innovación generan una ruptura fundamental frente a las trayectorias tecnológicas actuales, que para Rindova y Petkova (2007) implica que los usuarios no pueden aplicar los esquemas existentes para entender e interpretar la nueva tecnología, y para Christensen et al. (2009) que la evaluación de su desempeño no se pueda realizar bajo los atributos reconocidos por los usuarios. Es interesante resaltar que estas definiciones se centran en los usuarios y los mercados, sus percepciones, entendimientos, interpretaciones y atributos (que no incluyen las características físicas de la tecnología), dejando de lado la materialidad de la nueva tecnología.

\section{Cambio Institucional}

En un principio, el enfoque institucional de los estudios organizacionales centraron su atención en entender las organizaciones como instituciones embebidas, por sus miembros y líderes, de significado, valor y legitimidad (Selznick, 1957), donde el rol de estos actores era importante en los procesos de institucionalización (Battilana \& D'Aunno, 2009). Posteriormente, con el surgimiento del nuevo institucionalismo o neoinstitucionalismo, se entiende la institución como una estructura social que es creada por un conjunto de individuos u organizaciones, que colectivamente ejercen una acción u orientación para restringir un ambiente que será constantemente alterado en el tiempo (Barley \& Tolber, 1997; DiMaggio \& Powell, 1983; Meyer \& Rowan, 1977), y se enfocaron en el rol de estas estructuras formales en permitir o contener el comportamiento organizacional de manera que se explique la similaridad organizacional o efectos del isomorfismo, presentándose un cambio en el nivel de análisis que pasó de la organización al campo organizacional ${ }^{1}$, con énfasis en la difusión de normas y prácticas entre organizaciones, y donde los actores pasaron de tener una agencia importante, a tener un grado limitado de agencia humana, dando que el ambiente determina las respuestas de los individuos y organizaciones (Battilana \& D 'Aunno, 2009). En este sentido, el concepto de agencia humana es asociado a términos como motivación, voluntad, intencionalidad, interés, elección, autonomía y libertad (Batillana \& D'Aunno, 2009).

Tanto el viejo como el nuevo institucionalismo han presentado escepticismo por el modelo de actor racional (Powell \& DiMaggio, 1991), o modelo homo-economicus de la economía neoclásica, donde la agencia humana se ve reflejada en la capacidad que tienen los agentes de seleccionar la alternativa más eficiente, es decir, la alternativa que maximiza los resultados a partir de unos insumos, y donde se ignora el impacto que tiene el ambiente sobre los agentes, sus preferencias, decisiones y comportamientos (Battilana \& $\mathrm{D}^{\prime}$ Aunno, 2009).

En los últimos años han surgido nuevos enfoques institucionales a partir de la necesidad de contar con un mayor entendimiento sobre la desinstitucionalización y el cambio institucional, para dar respuesta a la pregunta de ¿cómo se crean nuevas instituciones o cómo cambian en el tiempo las instituciones existentes? (DiMaggio, 1988; Powell \& DiMaggio; 1991), que reincorpora el rol del actor y sus intereses dentro de la teoría institucional, llevando a repensar la relación entre agencia y estructura (Battilana \& D'Aunno, 2009; DiMaggio, 1988).

Por su parte, Oliver (1991, 1992), Greenwood et al. (2002), y Seo y Creed (2002), analizaron las fuentes del cambio institucional, tanto externas o ambiental como organizacionales, que pueden generar acciones de los actores que divergen de las instituciones actuales llevando a desinstitucionalizar las normas y prácticas existentes tanto a nivel organizacional como a nivel del entorno institucional o campo organizacional. Si una institución es un conjunto de prácticas, reglas, normas y rutinas que se han legitimado y tomado por sentadas 
por un conjunto de actores (Douglas, 1986; Seo \& Creed, 2002), entonces la disrupción institucional ocurre cuando estas características son cuestionadas por los actores y cuando uno o más de los mecanismos institucionales, que mantienen estas prácticas en su lugar, se desalinean o colapsan (Maguire \& Hardy, 2009), debido a que las instituciones no solo restringen la agencia humana sino que, precisamente, ellas son producto de la agencia humana (Powell \& DiMaggio, 1991).

Por otro lado, el estudio de la relación entre las instituciones de un campo organizacional y los actores que hacen parte de ellas, para entender el rol de los actores en la creación, transformar y mantenimiento las prácticas institucionalizadas (Lawrence \& Suddaby, 2006; Lawrence, Suddaby \& Leca, 2009; Lawrence, Suddaby \& Leca, 2011; Lawrence, Leca \& Zilber, 2013) tanto a nivel organizacional como de campo organizacional (Gawer \& Phillips, 2013), son resaltadas en el enfoque de trabajo institucional, el cual se centra en la "acción intencionada de individuos y organizaciones que buscan la creación, mantenimiento y disrupción de instituciones” (Lawrence \& Suddaby, 2006, p. 215).

\section{Cambio Tecnológico}

Zammuto, Griffith, Majchrzak, Dougherty y Faraj (2007)y Leonardi y Barley (2010)realizaron una revisión histórica de las investigaciones sobre tecnologías y su relación con las organizaciones. Estos autores identificaron dos perspectivas en las décadas de 1950 y 1960. Por un lado, una perspectiva desde la teoría de contingencia, donde se asigna a la tecnología un rol causal sobre la estructura organizacional (Zammuto et al., 2007, asumiendo la tecnología como un proceso de producción y como variable independiente, y la estructura organizacional como variable dependiente (Leonardi \& Barley, 2010. Por otro lado, identificaron investigaciones desde una perspectiva de sistemas socio-técnicos que, en oposición al determinismo tecnológico de la teoría de contingencia, se plantea una relación de influencia mutua entre la tecnología y la estructura social. A pesar de esto, los autores argumentan que los teóricos socio-técnicos de este periodo se centraban en la forma en que los sistemas sociales condicionaban las tecnologías y su uso, dejando de lado el rol de la tecnología en el cambio organizacional, generando un determinismo social (Leonardi \& Barley, 2010.

Leonardi y Barley (2010 argumentan que en la década de 1970, las investigaciones desde la perspectiva de sistemas socio-técnicos se detuvo, debido a que sus investigadores se centraron en la teoría de sistemas. Esto llevó a que la perspectiva de teoría de contingencia se convirtiera en la corriente dominante de investigación en tecnología (Leonardi \& Barley, 2010; Zammuto et al., 2007. A principios de la década de 1980 surge el denominado computerization movement, donde las investigaciones exploraban cómo tanto los individuos como las organizaciones respondían de forma diferente a los computadores. Por un lado, enfocado en cómo las actitudes e interpretaciones de las personas hacia las tecnologías afectan los patrones de su adopción y uso; por otro lado, enfocado en las organizaciones, especialmente sus políticas y cómo estas podrían conducir a dinámicas de implementación (Leonardi \& Barley, 2010.

En la década de 1980 las investigaciones en tecnología comenzaron a alejarse de los supuestos de la teoría de contingencia en rechazo del determinismo tecnológico (Zammuto et al., 2007; Leonardi \& Barley, 2010. Por un lado, desde la economía surgieron la escuela post-shumpeteriana que analizaban el desarrollo industrial y tecnológico enfatizando en las discontinuidades para explicar los patrones de la innovación en términos de los cambios en los paradigmas técnico-económicos (Freeman et al., 1982; Perez, 1983; Williams \& Edge, 1996. Algunos de los trabajos desarrollados desde esta perspectiva fueron criticados por generalizar el cambio tecnológico como motor del cambio socio-económico (Williams \& Edge, 1996. Otro grupo de economistas plantearon el modelo evolucionario de la innovación enfocándose no solo en los cambios radicales e incrementales como los post-shumpeterianos, sino que también se enfocaron en las características comportamentales de la firma y de los mercados, argumentando que los mercados son ambientes selectivos constituidos socialmente, lo cual puede privilegiar cierto tipo de tecnologías (Dosi, 1982; Nelson \& Winter, 
1982; Williams \& Edge, 1996). Algunas críticas a este modelo, principalmente al concepto de paradigmas y trayectorias de Dosi (1982), es que no es claro como al centrar la discusión a nivel de los paradigmas tecnológicos se logra una aproximación a los artefactos (Pinch \& Bijker, 1984).

Por otro lado, se argumentaba que las dinámicas sociales influyen en la adopción, implementación, uso y significado de la tecnología (Barley, 1986; Leonardi \& Barley, 2010). Desde las investigaciones de sistemas de información tecnológica se estudia la implementación de nuevas tecnologías basadas en computadores en contextos organizacionales establecidos, como por ejemplo el correo electrónico, las imágenes médicas, los groupware, los sistemas de soporte a decisiones, las herramientas de simulación digital, entre otros (Iacono \& Kling, 2001; Kling, 1991; Kling \& Iacono, 1988).

Desde la sociología también se encuentran diferentes vertientes de estudios sociales de la ciencia y la tecnología, centradas en su mayoría en estudiar la contribución de los procesos sociales en el desarrollo de nuevas tecnología, a partir de la inclusión de la materialidad de la tecnología (Callon, 1986; Latour, 1987; Pinch \& Bijker, 1984; Leonardi \& Barley, 2010) con el objetivo de "abrir la caja negra de la tecnología" (Pinch, 2008, p. 469), a la vez que pretenden responder a las críticas que hasta ese entonces se daba a los estudios de la sociología de la ciencia que se centran en el científico y la agencia humana (Pickering, 1995). En este sentido, autores como Callon (1986), Knorr-Cetina (1997) y Latour (2008) han planteado la disolución de los límites analíticos entre tecnologías y humanos con el planteamiento de una agencia simétrica, entendida como el relato de la acción, es decir, como una explicación de cómo se hace algo con incidencia, de alguna manera, en el estado de las cosas, a través de la Teoría de Actor-Red, que se enfoca en la descripción, no estática, de cómo emergen las redes de trabajo y como toman forma las interacciones entre los actores en un proceso de innovación (Latour, 2008).

Por su parte, Pickering (1995) argumenta que la agencia material es temporalmente emergente, es decir, "los contornos de la agencia material no se pueden conocer por adelantado, los científicos tiene que explorarlos de forma continua en su trabajo, los problemas van surgiendo y tienen que ser solucionados en el desarrollo" (Pickering, 1995, p. 564), adicionalmente plantea que la agencia material y humana están constitutivamente inmersas en la práctica, donde se encuentran en una dialéctica de resistencia y reacomodación, a lo que el autor denomina Mangle of practice. A su vez, autores como Pinch y Bijker (1984) expresando su crítica a la separación de la ciencia y la tecnología de los estudios hasta ese momento dominantes, argumentan que "en el análisis económico la innovación tecnológica todo lo que pueda influenciar la innovación es incluido, excepto cualquier discusión de la tecnología por sí misma” (Pinch \& Bijker, 1984, p. 404), planteando la integración del constructivismo social al estudio empírico de la ciencia y la tecnología, que denominan la construcción social de la tecnología. Posteriormente, Bijker (1995) propone el concepto de ensambles socio-técnicos, donde los hechos científicos y los artefactos tecnológicos deben ser entendidos como construcciones sociales, es decir, lo técnico es socialmente construido, y a su vez lo social es técnicamente construido.

Suchman (2007) argumenta que las dinámicas socio-materiales se encuentran en las prácticas diarias, donde se configuran y reconfiguran los roles de los actores por múltiples significados y materialidades que se fusionan juntos y co-emergen. Barad (2003) también centrada en las prácticas diarias, señala que "El lenguaje importa. El discurso importa. La cultura importa. Existe una sensación importante de que lo único que ya no importa es la materia" (Barad, 2003, p. 801), como crítica al determinismo social y al constructivismo social. Para esta autora los significados y las materialidades se encuentran representados [enacted] en las prácticas diarias (Barad, 2007), apoyando lo que denomina una metafísica performativa, que cambia el foco de análisis de los objetos independientes con propiedades y límites inherentes a las prácticas, es decir, formas de hacer/ actuar que realiza un fenómeno particular (Barad, 2003).

Por su lado, Latham y Sassen (2005) señalan el surgimiento de una nueva relación socio-tecnológica que identifican como las formaciones digitales, constructo desarrollado por los autores para designar tipos específicos de estructuras de información y comunicaciones, como pueden ser los mercados electrónicos y 
el desarrollo de software de código abierto [open source]. Los autores argumentan estas nuevas formaciones deben ser objeto de estudio, debido a que estas formas no existían en contextos anteriores y debido a la novedad de sus formas sociales que están reformando las relaciones sociales y constituyendo nuevos dominios sociales.

En la tabla 2 se presenta un resumen de los estudios encontrados que buscan vincular la materialidad y lo social en las investigaciones en tecnología y organizaciones.

TABLA 2

Resumen de estudios

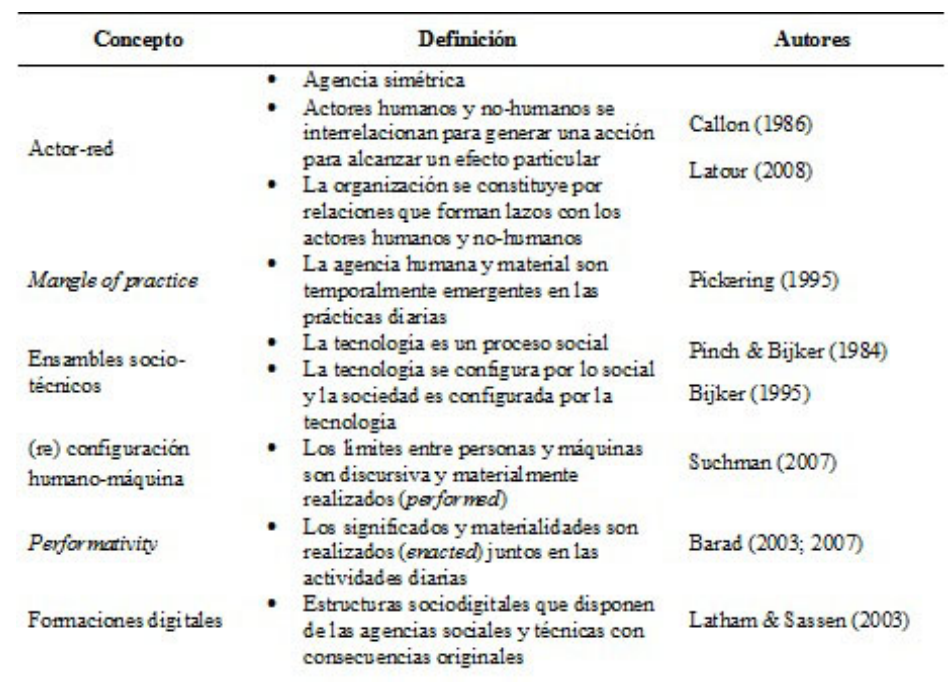

Fuente: adaptación de Orlikowski y Scott (2008, p. 458).

\section{Brechas en la literatura}

Después de la revisión de las teorías de cambio organizacional, institucional y tecnológico, se identificaron tres conceptos recurrentes en la literatura: nivel, agencia y tipo o modo de cambio. En la tabla 3 se presenta la definición de estos conceptos. 
TABLA 3

Definición de conceptos

\begin{tabular}{|c|c|}
\hline Concep tos & Definición \\
\hline \multirow[b]{2}{*}{$\begin{array}{l}\text { Niveldel } \\
\text { cambio }\end{array}$} & $\begin{array}{l}\text { Organizacional: entidad singular, es decir, individuo, grupo, de trabajo, } \\
\text { estrategia organizacional, programa, producto v organización (Van de Ven \& } \\
\text { Poole, 1995). }\end{array}$ \\
\hline & $\begin{array}{l}\text { Campo org anizacional: miltiples entidades u organizaciones que, en agregado, } \\
\text { constituyen un área reconocida de la vida institucional, como pueden ser los } \\
\text { proveedores, consumidores de recursos y prodvctos, agencias regulatorias y } \\
\text { otras organizaciones que producen productos o servicios similares (DiMag gio \& } \\
\text { Powell, 1983). }\end{array}$ \\
\hline \multirow{3}{*}{$\begin{array}{l}\text { Agencia del } \\
\text { cambio }\end{array}$} & $\begin{array}{l}\text { Estructura: ambiente que permite o contiene el comportamiento de indivióv os y } \\
\text { org anizacional al determinar sv respuesta, de manera que explica sv similarid ad } \\
\text { (Battilana \& D'Aunno, 2009). }\end{array}$ \\
\hline & $\begin{array}{l}\text { Agencia humana: motivación, voluntad, intencionalidad, interés, elección, } \\
\text { autonomia y libertad para la toma de decisiones y acciones de los actores } \\
\text { humanos (Battilana \& D Aunno, 2009). }\end{array}$ \\
\hline & $\begin{array}{l}\text { Agencia no-humana o material: describe la acción generada por un actor no- } \\
\text { humano, la cual es temporalmente emergente, es decir, no se pueden conocer } \\
\text { por adelantado (Pickering, 1995). }\end{array}$ \\
\hline \multirow{6}{*}{$\begin{array}{l}\text { Modo de } \\
\text { cambio }\end{array}$} & $\begin{array}{l}\text { Prescriptivo: secvencia de eventos de cambio que están determinados } \\
\text { previamente y son predecibles (Van de Ven \& Poole, 1995). }\end{array}$ \\
\hline & $\begin{array}{l}\text { Continuo: ocurre dentro de un sistema estable y en periodos de equilibrio } \\
\text { (Meyer et al., 1990; Nadler \& Tushman, 1995). }\end{array}$ \\
\hline & $\begin{array}{l}\text { Incremental: los esquemas disponibles pueden ser utilizados directamente o } \\
\text { requieren de pequeñas variaciones, que los usuarios podrian alcanzar mediante } \\
\text { extensiones del esquema (Rindova, \& Petkova, 2007), buscan mantener la } \\
\text { trayectoria existente del mercado, al mejora el desempeño de las } \\
\text { organizaciones, medido segun las dimensiones o los atributos que son } \\
\text { reconocidos por los usvarios (Christensen et al., 2009). }\end{array}$ \\
\hline & $\begin{array}{l}\text { Constructivista progresión que es construida y emerge mientras el proceso de } \\
\text { cambio se despliega, generando cambios que rompen con los supvestos y } \\
\text { marcos utilizados hasta el momento (V an de Ven \& Poole, 1995). }\end{array}$ \\
\hline & $\begin{array}{l}\text { Discontinuo: genera transformación de 1as propiedades fundamentales del } \\
\text { sistema durante periodos de disupción (Meyer et al., 1990; Nadler \& Tushman, } \\
\text { 1995). }\end{array}$ \\
\hline & $\begin{array}{l}\text { Disruptivo: genera una ruptura fundamental frente a las trayectorias } \\
\text { tecnológicas actuales, lo que implica que los usuarios no pueden aplicar los } \\
\text { esquemas existentes (Rindova y Petkova, 2007), y la evaluación no cumplen } \\
\text { con los parámetros de desempeño actuales del mercado (Christensen et al., } \\
\text { 2009). }\end{array}$ \\
\hline
\end{tabular}

Fuente: elaboración propia.

En la tabla 4 se presentan las complementariedades y diferencias entre las teorías revisadas, las cuales se categorizaron en Cambio Organizacional, Cambio Institucional y Cambio Tecnológico, frente a tres elementos que se encontraron de forma recurrente en la literatura: nivel, agencia y tipo o modo de cambio.

TABLA 4

Complementariedades y diferencias teóricas

\begin{tabular}{|c|c|c|c|c|c|c|c|}
\hline \multirow[b]{3}{*}{$\begin{array}{l}\text { Cambio } \\
\text { Organizacional }\end{array}$} & \multicolumn{2}{|c|}{ Nivel } & \multicolumn{3}{|c|}{ Agencia } & \multicolumn{2}{|c|}{ Modo de Cambio } \\
\hline & aganización & $\begin{array}{c}\text { Campo } \\
\text { aganizocional }\end{array}$ & Humana & $\begin{array}{c}\text { No- } \\
\text { numana }\end{array}$ & Estuaura & continuo & Discontinuo \\
\hline & & & & & & & \\
\hline Cislo de Vida & Si & No & No & No & $\mathrm{Si}$ & $\mathrm{Si}$ & No \\
\hline Teleología & $\mathrm{Si}$ & No & $\mathrm{Si}$ & No & No & No & $\mathrm{Si}$ \\
\hline Dialéctica & No & $\mathrm{Si}$ & $\mathrm{Si}$ & No & No & No & $\mathrm{Si}$ \\
\hline Evolución & No & $\mathrm{Si}$ & No & No & $\mathrm{Si}$ & $\mathrm{Si}$ & No \\
\hline \multicolumn{8}{|l|}{$\begin{array}{l}\text { Cambio } \\
\text { Institucional }\end{array}$} \\
\hline Neo-institucional & No & $\mathrm{Si}$ & No & No & $\mathrm{Si}$ & $\mathrm{Si}$ & $\mathrm{NE}^{*}$ \\
\hline Trabajo Instituciona1 & $\mathrm{Si}$ & $\mathrm{Si}$ & $\mathrm{Si}$ & No & $\mathrm{Si}$ & $\mathrm{Si}$ & $\mathrm{Si}$ \\
\hline \multicolumn{8}{|l|}{$\begin{array}{l}\text { Cambio } \\
\text { Tecnológico }\end{array}$} \\
\hline Contingencia & $\mathrm{Si}$ & $\mathrm{NE}^{*}$ & No & $\mathrm{Si}$ & No & $\mathrm{Si}$ & No \\
\hline Constructivista & Si & No & $\mathrm{Si}$ & No & No & $\mathrm{NE}^{*}$ & $\mathrm{NE}^{*}$ \\
\hline Teoria Actor-Red & $\mathrm{Si}$ & $\mathrm{Si}$ & $\mathrm{Si}$ & $\mathrm{Si}$ & No & No & $\mathrm{Si}$ \\
\hline
\end{tabular}

* NE: no es explícito.

Fuente: elaboración propia.

De esta comparación se pueden resaltar que las teorías de ciclo de vida y evolución dentro del cambio organizacional, se pueden categorizar como continuos y centradas en la estructura, debido a que se presenta una concepción pasiva de los actores organizacionales en favor de fuerzas abstractas y sin intencionalidad como son el mercado y las fuerzas ambientales. En este sentido, estos conjuntos de teorías se relacionan con la teoría de contingencia y neo-institucional, donde la agencia generadora del cambio se encuentra por fuera de las organizaciones, en el primer caso, siendo la tecnología la fuerza determinante de los cambios 
organizacionales, y en el segundo, siendo las instituciones las estructuras que permiten o restringe los cambios en las organizaciones.

Dentro de las teorías de cambio tecnológico se resalta que la teoría de contingencia presenta la tecnología como una variable independiente o moderadora de los cambios a nivel de la organización (Orlikowski \& Scott, 2008), es decir, se puede categorizar dentro de la agencia no-humana, minimizando el rol de la historia, el contexto social y la agencia humana en la conformación de la producción, uso y cambio tecnológico (Orlikowski, 2010). Por otro lado, las teorías agrupadas dentro del constructivismo, a pesar de plantear una interdependencia entre los humanos y/o organizaciones y la tecnología, sigue centrando la relación sobre la agencia humana (Orlikowski, 2010). Adicionalmente, a pesar de plantear que las tecnologías son socialmente definidas y producidas, este planteamiento no genera claridad si esta construcción social es continua o discontinua.

En el cambio institucional, específicamente en el trabajo institucional, se puede explicar tanto el cambio a nivel organizacional como del campo organizacional. Pero al igual que la perspectiva constructivista de cambio tecnológico, se centra en la agencia humana dejando por fuera el rol de la materialidad de las tecnologías en los procesos institucionales (Dover \& Lawrence, 2010; Jones \& Massa, 2013; Raviola \& Norbäck, 2013; Lawrence et al., 2013; Montero \& Nicolini, 2014. Los mecanismos de trabajo institucional pueden ayudar a dar cuenta de los modos de cambio continuo como es el mantenimiento de las instituciones, vinculándose a la teoría de ciclo de vida y evolución, pero a diferencia de estas teorías, centrándose en la agencia humana. Por otro lado, el trabajo institucional ayuda también a entender los cambios discontinuos como son la creación y disrupción de instituciones, relacionándose con las teorías teleológica y dialéctica. Desde la teoría teleológica, se plantea que el cambio se da a partir de los aprendizajes de los actores, que pueden modificar sus objetivos y cursos de acción, mientras que desde la teoría dialéctica, el cambio se da por un juego de poder entre entidades que se oponen, dando en ambos casos un papel central a la agencia humana (Van de Ven \& Poole, 1995.

Es interesante como este proceso de cambio planteado desde la teoría dialéctica, el cual emerge desde el conflicto entre entidades con una agencia humana, que se presentan como tesis, antitesis y síntesis (Van de Ven \& Poole, 1995, se puede asemejar a la teoría Actor-red y su proceso de traducción que presenta la trayectoria de una tecnología, como un conjunto de interrelaciones que pasan por una serie de desvíos (oposición y composición (apoyo (Latour, 2008. Pero a diferencia de la teoría dialéctica, la teoría Actor-red, integra en su análisis la agencia que surge de la interrelación entre actores humanos y no-humanos.

Finalmente, se observa como desde la teoría Actor-red se puede analizar la interrelación de la agencia humana y no-humana, tanto a nivel organizacional como de campo organizacional, principalmente centrándose en el modo de cambio discontinuo, ya que en el estudio de las innovaciones, los objetos generan procesos de reasociación y reensamblaje que permiten rastrear las nuevas asociaciones de los actores y el diseño de nuevos ensamblados, debido a que se mantienen por más tiempo como actores visibles, distribuidos, referidos, antes de volverse invisibles y asociales (Latour, 2008, debido que en el momento de institucionalizarse se comienzan a dar por sentado.

\section{RELACIÓN CAMBIO TECNOLÓGICO, ORGANIZACIONAL E INSTITUCIONAL}

Adicional al análisis de las teorías, de sus brechas y complementariedades, se realiza un análisis más detallado de las teorías revisadas y se identifica la relación entre cambio organizacional, cambio tecnológico y cambio institucional. 


\section{Relación cambio tecnológico y organizacional}

Autores como Barley (1986), Zammuto et al. (2007), Leonardi y Barley (2008, 2010), Orlikowsky y Scott (2008), y Orlikowsky (2010) argumentan que la tecnología ha sido caracterizada dentro de los estudios organizacionales como objeto físico o producto social. Barley (1986) por ejemplo, argumenta que muchas de estas investigaciones resultan en un determinismo, entendido como que las acciones humanas son causadas por fuerzas tecnológicas, culturales y otras antes de, externas a, e independientes de nuestro comportamiento (Leonardi \& Barley, 2008), por lo que los estudios que se han enfocado en los aspectos físicos de la tecnología entran en un materialismo que a menudo resulta en un determinismo tecnológico, donde se plantea que los efectos de la tecnología en la vida social son determinados e inevitables. Por otro lado, los estudios que se han enfocado en la tecnología como una producción social han llevado a un exceso de confianza en la cultura como principal impulsor, resultando en una forma de determinismo social (Barley, 1986). Zammuto et al. (2007) plantean que el enfoque central de las investigaciones organizacionales sobre la tecnología ha pasado por la teoría de la contingencia, a teorías como la institucional, de ecología de poblaciones, y dependencia de recursos, pero que el problema con la literatura actual es la falta de atención en la cambiante relación entre tecnología y organización.

Leonardi y Barley (2008), reconociendo la ausencia del rol de la materialidad en los estudios de tecnología y organizaciones en los últimos años, plantean una reconciliación entre el materialismo y el voluntarismo. En este sentido, Leonardi y Barley (2008) argumentan que el materialismo es importante para las teorías de tecnología y organizaciones porque las propiedades materiales de los artefactos son precisamente recursos tangibles que proveen a la gente con la habilidad de hacer cosas ya conocidas pero de nuevas maneras, o de hacer cosas que antes no podían hacer. Estos autores aclaran que ser materialista no necesariamente conlleva al determinismo y proponen que para integrar la materialidad con una visión voluntarista se requiere que los investigadores identifiquen formas específicas en las que las características particulares de los artefactos se entrelazan con las prácticas sociales de las personas en su trabajo.

A pesar de que Leonardi y Barley (2008) reconocen la necesidad de integrar la materialidad a los estudios tecnológicos y organizacionales, para estos autores la agencia sigue centrándose en los humanos, ya que definen que ser materialista es plantear que la acción humana surge de causas físicas y contextos como el geográfico, biológico, clima y tecnología, mientras que ser voluntarista es argumentar que los humanos tenemos agencia (libre albedrío) y que este puede dar forma a su ambiente para alcanzar sus intereses y objetivos.

Por su parte, Orlikowski y Scott (2008), a partir de un análisis de los artículos publicados en las revistas más relevantes en temas de Management, clasificaron la literatura de tecnología en organizaciones en dos corrientes de investigación, como una entidad discreta y como unidades mutuamente dependientes, relacionando la primera corriente con la Teoría de Contingencia y la segunda corriente con la Teoría de Estructuración.

Usualmente la tecnología se trata como un elemento exógeno; en estudios empíricos se ha manejado como una variable independiente en unos casos y, en otros, como moderadora, causando o moderando la relación con varios aspectos de la organización (Orlikowski \& Scott, 2008). En este marco, la tecnología es tratada como un elemento material y determinante causal de los elementos organizacionales, haciendo parte de los estudios en Management del enfoque de la Teoría de la Contingencia (Klein, 2006 en Orlikowski, 2010). La tecnología como unidades mutuamente dependientes es entendida como un proceso emergente, que surge de la interacción entre las elecciones humanas, acciones e historia social y contexto institucional. Un elemento central de este marco es que el entendimiento de la tecnología no es fijo o universal, sino que surge de un contexto particular, a pesar de que pueda tratarse del despliegue de tecnologías idénticas, que plantea que la incertidumbre y complejidad tecnológica son construcciones sociales que varían de un contexto a otro (Barley, 1986; Orlikowski, 2010). 
Entre los problemas que identifica Orlikowski (2010) de estas dos corrientes de investigación desde la literatura del management, se encuentra por un lado que, al enfocarse en la tecnología como una fuerza exógena, causante u ocasionadora de efectos o cambios organizacionales, se minimiza el rol de la historia, del contexto social y de la agencia humana, en la conformación de la producción, uso y cambio tecnológico. Adicionalmente, esta corriente ha sido criticada por no tener en cuenta o reducir la dinámica y materialidad situada que constituyen a las tecnologías. Por otro lado, a pesar que la segunda corriente se identifique como de interrelación, la tecnología es entendida como un artefacto material que es socialmente definido y producido, por lo tanto, es relevante solo con relación a las personas que interactúan con ellos, dando un rol más preponderante a la agencia humana dentro del cambio tecnológico y minimizando el rol de la tecnología y en especial de sus características físicas.

Desde las vertientes de estudios sociales de la ciencia y la tecnología de la sociología, también se han centrado en la contribución de los procesos sociales en el desarrollo de nueva tecnología, a partir de la inclusión de la materialidad de la tecnología (Callon, 1986; Latour, 1987; Pinch \& Bijker, 1984; Leonardi \& Barley, 2010). Pero muchos de estos autores caen de nuevo en la división analítica que pretenden subsanar: agencia humana y material (Pickering, 1995), ensambles socio-técnicos, es decir, lo técnico es socialmente construido y a su vez que lo social es técnicamente construido (Bijker, 1995), agencias sociales y técnicas (Latham \& Sassen, 2005).

Un ejemplo de la relación entre el cambio organizacional y tecnológico a partir de la teoría actorred es la investigación de Powell y Grodal (2006, quienes argumentan que los procesos asociados a la innovación a través de la incorporación de tecnologías (sistemas de información, herramientas técnicas, equipos y conocimiento en las organizaciones toman lugar en redes de intercambio de información, sociales y económicas entre la organización y su entorno a través de complejas relaciones que afectan, por una parte, el desempeño productivo de la organización y, por otra, su funcionamiento, por ejemplo a formas más flexibles de la organización y la redefinición de estrategias de la empresa y de los procesos de toma de decisiones. Por su complejidad y sus resultados estos procesos han generado la necesidad de abordar estudios desde las dinámicas de cambios en términos de la colaboración con diferentes actores, la intensidad de dichas colaboraciones, de la coordinación y estructura, el aprendizaje y los costos de estas relaciones. Para abordar la diversidad de actores y vínculos, y la complejidad del papel de los vínculos de relación, se han utilizado las redes de trabajo (Powell y Grodal, 2006, las cuales no sólo se han analizado, en estudios transeccionales, sino también en su dinámica evolutiva que explique retardos y limitaciones en los procesos de implementación (apropiación, uso e interpretación de las relaciones.

Algunas relaciones en procesos de implementación y cambio requieren además de los vínculos personapersona, relaciones de individuos con otros tipos de actores no solamente formales sino informales, que examinen entrelazamientos con estrategias, dinámicas y trayectorias de los proveedores y usuarios (Powell \& Grodal, 2006, en donde muchas veces las diferencias y dependencias de dichas relaciones son poco claras con aspectos interpretativos diferenciales que generan aprendizajes, impactos y costos relacionados, con los conocimientos que están siendo usados y transformados, dependiendo de las características de los adoptadores y efectividad de los resultados (Van de Ven, 2007.

Desde la perspectiva de redes de actores, basados en la teoría de actor-red (Powell \& Grodal, 2006 se estudian los vínculos de actores de las organizaciones y sus entornos; sin embargo no sólo humanos sino también artefactos, por ejemplo, elementos técnicos, documentaciones, directrices, recursos de procedimientos formales e informales de relación, que en su conjunto se denominan actantes, que metodológicamente se relacionan a través de procesos de traducción, que más allá de los procesos de interacción lo que se identifican son recombinaciones novedosas de resultados imprevistos de decisiones, conocimientos, o soluciones de problemas existentes, tal como lo plantea genéricamente Nelson y Winter $(1982$. 
En conclusión, la relación entre tecnología y organizaciones ha sido ampliamente estudiada desde diversas disciplinas. En la figura 2 se presenta un resumen de las relaciones presentadas en esta literatura.

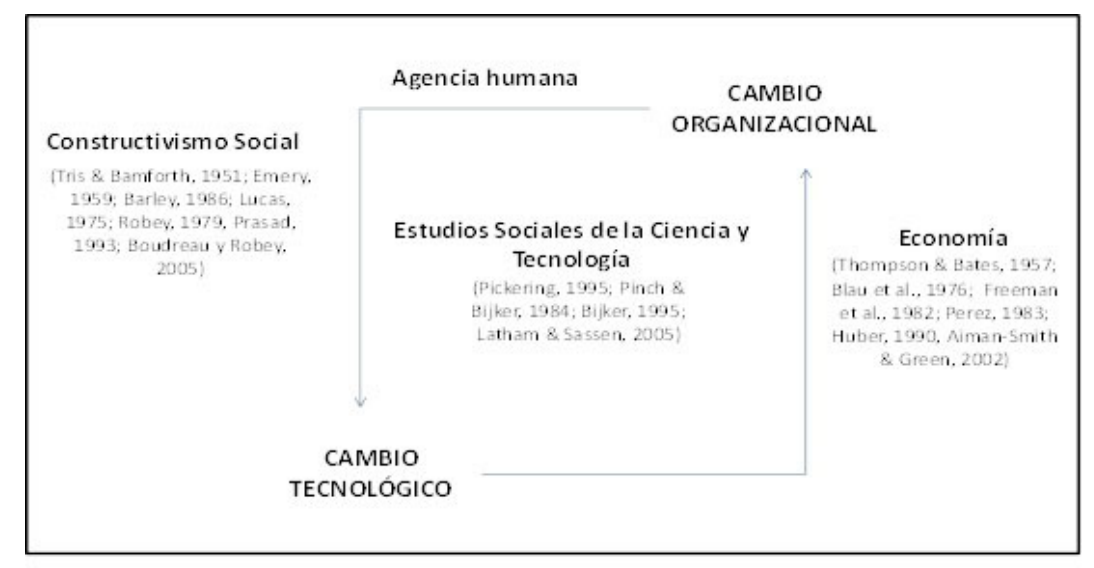

FIGURA 2

Relación cambio tecnológico y organizacional desde la literatura

Fuente: elaboración propia.

\section{Relación cambio institucional y organizacional}

Las fuentes del cambio institucional, tanto externas o ambientales como organizacionales, que pueden generar disrupción o desinstitucionalizar las normas y prácticas existentes a nivel organizacional como del entorno institucional o campo organizacional han sido estudiadas por diversos autores (Oliver, 1991, 1992; Greenwood et al., 2002; Seo \& Creed, 2002).

Los cambios externos pueden ser ocasionados por disrupciones que ocurren en los sistemas vecinos que desestabilizan las reglas y entendimientos existentes a nivel social, tecnológico o regulatorio (Greenwood et al., 2002; Scott, 2008). Por otro lado, las fuentes internas de cambio se pueden dar por las brechas o discrepancias entre los sistemas macro y las actividades micro de los actores en respuesta a circunstancias locales, más específicamente, la desinstitucionalización se refiere a los procesos por los cuales la legitimidad de una práctica organizacional institucionalizada es debilitada y remplazada, como un resultado de las respuestas proactivas o pasivas de las organizaciones a los cambios internos y eventos externos (Oliver, 1991; Pache \& Santos, 2010).

Oliver (1992) identifica tres fuentes principales de presiones sobre las normas o prácticas institucionalizadas de la organización, que pueden ser tanto externas como internas a la organización, estas son: funcionales, políticas y sociales. Las presiones funcionales son aquellas que surgen cuando se perciben problemas en el desempeño o en la utilidad asociada con las prácticas institucionalizadas, y pueden estar relacionadas a cambios ambientales como, por ejemplo, la rivalidad por recursos (Dancin, Goodstein \& Scott, 2002). Por otro lado, las presiones políticas son el resultado de un cambio de intereses y distribución subyacente del poder, los cuales han soportado y legitimado los arreglos institucionales existentes; también se generan por el incremento en la presión para la adopción de prácticas innovadoras al interior de la organización (Oliver, 1992). Finalmente, la autora plantea que el cambio institucional puede ser influenciado por presiones sociales asociadas con la diferenciación de grupos, la existencia de heterogeneidades divergentes o creencias y prácticas discordantes, y cambios estructurales tanto al exterior como interior de la organización (Oliver, 1992).

Adicionalmente, Oliver (1991) y Pache y Santos (2010) han planteado modelos de respuestas organizacionales hacia las demandas institucionales conflictivas que puede llevar al cambio de prácticas organizacionales, resaltando el rol de los intereses y la agencia en la conformación de la acción. Agencia que se evidencia en las respuestas estratégicas de los actores organizacionales (Oliver, 1991; Scott, 2008). 
A nivel del ambiente institucional o campo organizacional, Seo y Creed (2002) plantean cuatro fuentes de contradicción que pueden resultar en cambios institucionales. Primero, los arreglos institucionales que tienen como objeto incrementar la legitimidad en el corto plazo previenen la continua búsqueda de soluciones óptimamente eficientes, incrementando las ineficiencias técnicas en el largo plazo. Segundo, el isomorfismo institucional, que incrementa la legitimidad, es un movimiento de adaptación para lograr la supervivencia, pero en el largo plazo este tipo de movimientos disminuye la capacidad de adaptabilidad. Tercero, la conformidad a un conjunto de arreglos institucionales de un nivel o sector particular, puede causar conflictos o inconsistencias con los arreglos institucionales de diferentes niveles o sectores. Finalmente, los autores manifiestan que los arreglos institucionales probablemente reflejan las ideas y objetivos de los actores más poderosos, pero que es poco probable que la formación y reproducción de estos arreglos satisfagan los intereses divergentes de todos los actores, por lo que esta divergencia de intereses y el poder puede ser explorada en estudios de cambio institucional.

Por otro lado, el Trabajo Institucional estudia la relación entre las instituciones de un campo organizacional y los actores que hacen parte de ellas, para entender el cambio institucional desde el rol de los actores en crear, transformar y mantener las prácticas institucionalizadas (Lawrence \& Suddaby, 2006; Lawrence, Suddaby \& Leca, 2009; Lawrence, Suddaby \& Leca, 2011; Lawrence, Leca \& Zilber, 2013), que se definen como las prácticas que se establecen en los ambientes institucionalizados, y que las organizaciones adoptan, difundiéndose tan ampliamente que se dan por sentadas, y por lo tanto son resistentes al cambio (Battilana \& D'Aunno, 2009).

En la figura 3 se presenta un resumen de la relación entre cambio institucional y organización identificada en la literatura.

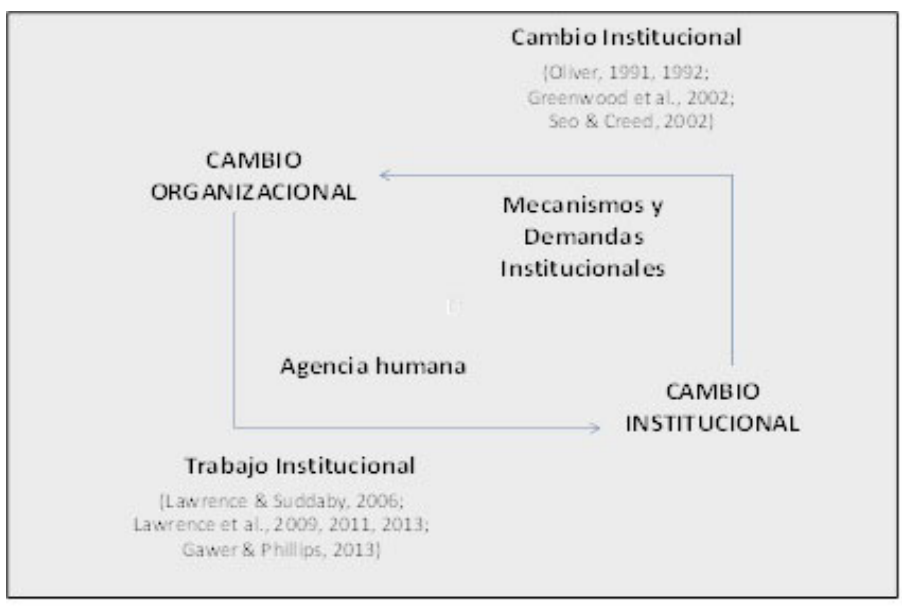

FIGURA 3

Relación cambio institucional y organizacional desde la literatura

Fuente: elaboración propia.

\section{APROXIMACIÓN AL CAMBIO ORGANIZACIONAL, TECNOLÓGICO E INSTITUCIONAL DESDE LA TEORÍA DE ACTOR-RED Y EL TRABAJO INSTITUCIONAL}

En el apartado anterior se hace evidente la importancia de la relación entre los cambios organizacionales, tecnológicos, e institucionales, pero a pesar de su importancia esta relación ha sido estudiada de forma parcial.

En este sentido, se seleccionan la Teoría Actor-red y el enfoque de trabajo institucional (Lawrence \& Suddaby, 2006; Lawrence et al., 2009), como las teorías base que pueden vincular la relación entre cambio organizacional, tecnológico e institucional, al incluir los conceptos de actor no-humano, agencia distribuida, formas de trabajo institucional y mecanismos institucionales; y complementar su análisis, eliminando los 
límites analíticos entre tecnologías y humanos (Callon, 1986; Knorr-Cetina, 1997; Latour, 2008), lo que permite estudiar la acción (formas de trabajo institucional) que se genera de la interrelación de actores humanos y no-humanos, por lo que no distingue lo humano de lo no-humano para designar la agencia, definiendo la agencia como el relato de la acción, es decir, es una explicación de cómo se hace algo con incidencia, de alguna manera, en el estado de las cosas (Latour, 2008).

A pesar de que esta aproximación no es nueva, son pocos los artículos que han integrado dentro de sus investigaciones, conceptos y metodologías que vinculan la Teoría Actor-red -TAR-y la Teoría Institucional. Por ejemplo, Tryggestad y Georg (2011) y Waldorff (2013) utilizan la teoría actor-red, específicamente las prácticas materiales y la traducción, para entender a nivel micro las prácticas, actores, agencia y significados que afectan las lógicas institucionales. Mientras que Yang y Wang (2013) utilizan la TAR para identificar los actores claves en las diferentes etapas de la evolución de un mercado electrónico y los vinculan con los mecanismos institucionales (cognitivo-cultura, regulatorio, normativo), proponiendo la inclusión de un nuevo mecanismo institucional denominado tecnología. Finalmente, Beunza y Ferraro (2019) utilizan el concepto de traducción y lo vinculan al trabajo institucional, para explorar el diseño de micro-políticas, promoción y uso de dispositivos, proponiendo un nuevo concepto que es performative work.

Para comprender los conceptos relacionados tanto en el trabajo institucional como en la teoría actor-red, a continuación se presenta un resumen de sus principales elementos.

\section{Trabajo Institucional}

Las instituciones como construcciones sociales requieren una interacción permanente entre sus actores para mantener la estabilidad institucional (Berger \& Luckman, 1967) mediante el apoyo, corrección y recreación de los mecanismos que garanticen su cumplimiento social (Lawrence \& Suddaby, 2006), aceptabilidad y credibilidad, es decir legitimidad, que Suchman (1995, p. 574) define como "la percepción generalizada o supuesto que las acciones de una entidad son deseables, correctas, o apropiadas dentro de un sistema social de normas, valores, creencias y definiciones". Estos mecanismos institucionales son el resultado de una serie de acciones, a través de las cuales, las instituciones afectan a las organizaciones y, a su vez, las organizaciones afectan los arreglos institucionales en los que operan (Greenwood \& Suddaby, 2006; Lawrence et al., 2009).

Los mecanismos institucionales se pueden dividir en elementos regulatorios, normativos y cognitivosculturales, que proveen estabilidad y significado a la vida social (Maguire \& Hardy, 2009; Scott, 2001). El elemento regulatorio se refiere a la autoridad que ciertos actores utilizan para limitar el comportamiento de los actores (Caronna, 2004 en Maguire \& Hardy, 2009) de un campo organizacional, y que además comparten un sistema de significados, e interactúan frecuentemente entre ellos (Hoffman, 1999; Scott, 1994-1995 en Galvin, 2002). Este elemento regulatorio requiere la habilidad para establecer reglas, y políticas de conformidad y, si es necesario, coaccionar su cumplimiento (Scott, 2001 en Maguire \& Hardy, 2009), a través de las cuales se establece qué tipo de actores pueden participar, cuáles son sus derechos, y la legitimidad de sus acciones (Meyer, Boli \& Thomas, 1987). Por otro lado, el elemento normativo influencia el comportamiento, al definir qué es lo apropiado o esperado en una situación social dada (Wicks, 2001 en Maguire \& Hardy, 2009), más específicamente consiste en los valores y normas que producen la conformidad (Caronna, 2004 en Maguire \& Hardy, 2009) como resultado de una expectativa social y obligaciones morales. Finalmente, el elemento cognitivo se basa en las concepciones compartidas que constituyen la naturaleza social de la realidad (Scott, 2001 en Maguire \& Hardy, 2009). Los elementos cognitivo-cultural incorporan símbolos (palabras, signos, gestos, imágenes) como reglas culturales, que promueven los significados compartidos y su internalización en el comportamiento (Hoffman, 1999).

En este sentido, el estudio del trabajo institucional busca reorientar la atención de la relación entre las organizaciones y su campo organizacional, para entender cómo las acciones de los actores afectan las instituciones (Lawrence, Suddaby \& Leca, 2009), tanto a nivel organizacional como de campo organizacional 
(Gawer \& Phillips, 2013), resignificando la agencia humana en la relación con las instituciones, al promover el entendimiento de cómo los actores pueden tanto reproducir como desafiar las instituciones, ya que las instituciones se consideran como el producto -intencional o no- de acciones específicas tomadas por actores para reproducir, alterar o destruir las instituciones (Lawrence \& Suddaby, 2006), a partir de la modificación de los mecanismos institucionales que las soportan (ver figura 4).

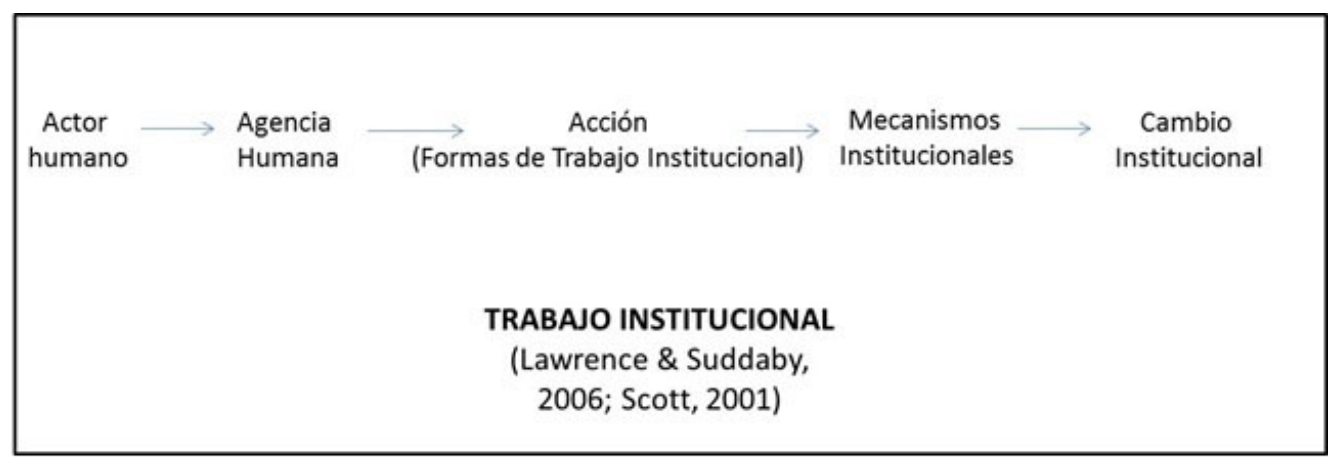

\section{FIGURA 4 \\ Conceptos teóricos desde el Trabajo Institucional \\ Fuente: elaboración propia.}

La agencia ha sido definida como la habilidad de los actores para operar, independientemente de las restricciones determinantes de la estructura social (Calhoun, 2002 en Battilana \& D'Aunno, 2009), definición que para Battilana y D 'Aunno (2009) puede llevar a diferentes interpretaciones, por un lado, se puede plantear que los actores despliegan su agencia cuando pueden tomar decisiones que van en contra de las restricciones de la estructura social, independientemente de si alteran o no la estructura social (Battilana \& D'Aunno, 2009); por otro lado, se puede argumentar que los actores despliegan su agencia solo cuando alteran los sistemas existentes y las reglas (Scott et al., 2000).

Battilana y D'Aunno (2009) complementan esta definición argumentando que la agencia humana no es un atributo constante, sino que varía dependiendo del contexto en el que los actores se encuentren incrustados y que puede cambiar en el tiempo. Además, plantean que la agencia es el compromiso del actor con el mundo social que, a través de la interacción de hábito, imaginación y juicio, puede reproducir y transformar las estructuras del ambiente.

El hábito se relaciona con la reactivación selectiva de los patrones pasados a través de la acción de los actores, ya que los actores pueden participar en rutinas habitualizantes y prácticas que reproducen instituciones, pero a menudo lo hacen a conciencia y con un propósito, más que simplemente actuar como autómatas institucionales (Emirbayer \& Mische, 1998 en Battilana \& D'Aunno, 2009). Por su parte, la imaginación involucra un compromiso con el futuro a través de posibles trayectorias de acción (Emirbayer \& Mische, 1998 en Battilana \& D'Aunno, 2009). Finalmente, el juicio corresponde a la capacidad del actor de evaluar las trayectorias alternativas de acción en respuesta a las demandas, dilemas y ambigüedades emergentes de las situaciones presentes (Emirbayer \& Mische, 1998 en Battilana \& D'Aunno, 2009).

Otro de los elementos a resaltar de la perspectiva de trabajo institucional, es su fundamentación en la sociología de la práctica (Bourdieu, 1977; 1993; de Certeau, 1984; Giddens, 1984 en Lawrence \& Suddaby, 2006), para conceptualizar la interrelación entre los actores y sus ambientes institucionalizados.

A pesar de esto, se identifica que al definir el trabajo institucional como una "acción intencionada de individuos y organizaciones que buscan la creación, mantenimiento y disrupción de instituciones" (Lawrence \& Suddaby, 2006, p. 215), estas investigaciones han dejado por fuera el rol de la materialidad en los procesos institucionales, al centrarse solo en la agencia humana. 


\section{Teoría Actor-red}

Para Latour (2008) el término social es utilizado para fenómenos con una estabilidad, que ya están ensamblados, por lo cual este autor replantea esta definición como movimiento de reasociación y reesamblado, que permite rastrear las nuevas asociaciones de los actores y el diseño de nuevos ensambles (ver figura 5). Así, los arreglos sociales son fenómenos sociales que se ejercen y sostienen en un espacio y tiempo a través de los esfuerzos conjuntos entre humanos y no-humanos.

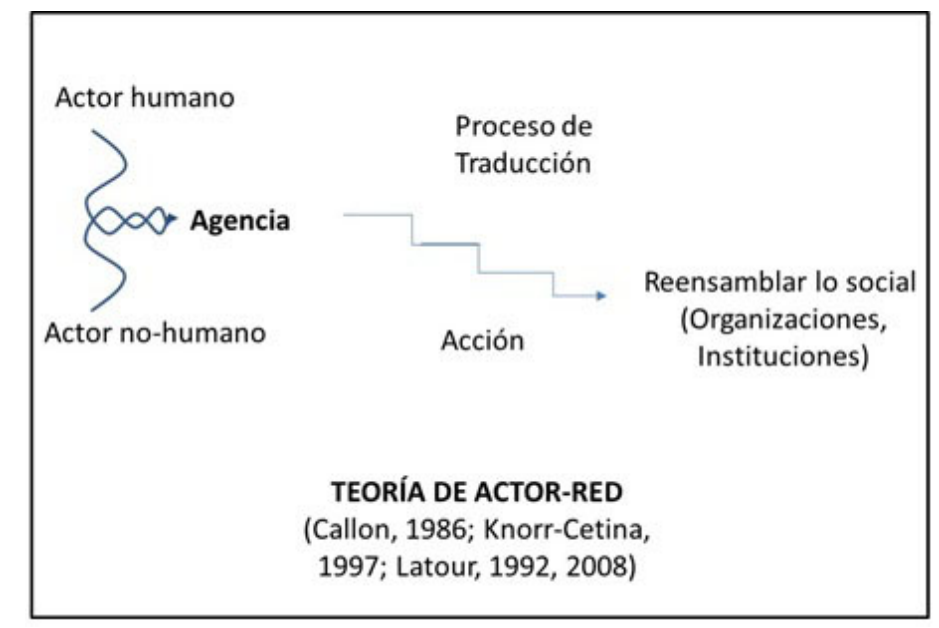

FIGURA 5

Conceptos teóricos desde la TAR

Fuente: elaboración propia.

Latour (1992) argumenta que una forma de resolver la dicotomía entre el determinismo tecnológico y el constructivismo social en los estudios tecnológicos es la Teoría Actor-red -TAR-, que se encuentra alineada con las afirmaciones social constructivistas en el sentido que los sistemas socio-tecnológicos son desarrollados a través de las negociaciones entre personas, instituciones y organizaciones, pero al mismo tiempo se diferencia de esta corriente al argumentar que los artefactos también son parte de estas negociaciones. De esta manera la TAR se enfoca en la descripción no estática de cómo emergen las redes de trabajo y cómo las interacciones entre los actores toman forma en un proceso de innovación (Latour, 2008). Igualmente, estudia cómo estas redes de trabajo se convierten en las llamadas "cajas negras", que implican que las interacciones humanas y no-humanas se dan por sentado (Sayes, 2014), permitiendo identificar la creación de nuevas prácticas e instituciones. Latour (1987) argumenta que la innovación tiene que ser estudiada en acción, lo cual permite enfocarse en las dinámicas y no en las estabilidades de las relaciones. En este sentido, la innovación es el resultado de una formación dinámica de alianzas, donde las cosas materiales también desempeñan un papel (Sayes, 2014).

Latour (2008) afirma que la TAR cuenta con unas particularidades como son: i) la asignación de capacidad de agencia a los actores no-humanos, es decir, estos actores también son participantes en el curso de la acción (Callon, 1986; Law, 1992; Latour, 2008) pero a diferencia de las acciones humanas, no se realizan bajo pleno control de la conciencia, por lo tanto, no buscan de forma deliberada el cambio organizacional, ni institucional; ii) lo social no es estable, por lo tanto, los actores no son definidos y analizados en un conjunto estable de relaciones, sino en un contexto dinámico (Latour, 1987); iii) el estudio apunta a reensamblar lo social. Desde esta perspectiva teórica, la definición de social se entiende como un movimiento de reasociación y reensamblado, que permite rastrear las nuevas asociaciones de los actores y el diseño de nuevos ensamblados (Latour, 2008).

Latour (2015) argumenta que la dificultad que se tiene para incluir los actores no-humanos y sus interrelaciones con los actores humanos en la creación de nuevas prácticas e instituciones, es que estos artefactos tecnológicos o actores no-humanos son invisibles para nosotros porque los vemos como piezas de 
cosas, y olvidamos su temporalidad, su historia y por lo tanto no sabemos cómo representar sus movimientos en el tiempo, para lo cual recomienda considerar la tecnología como proyecto y no como objeto.

Para analizar este ensamblaje se utiliza el concepto de traducción, que describe el movimiento de ideas y prácticas a través de los límites sociales (Callon \& Latour, 1981; Latour, 1986; Lawrence, 2017), enfocándose en los esfuerzos de los actores para dar forma a las ideas y prácticas para alcanzar su legitimidad, en algunos casos, desafiando las actuales (Lawrence, 2017). En este mismo sentido Law (1992) argumenta que la traducción es un concepto central de la teoría de actor-red, que se preocupa por cómo los actores y las organizaciones se movilizan, yuxtaponen y mantienen unidos las partes de los ensamblajes en los que se encuentran asociados, así como estos actores pueden convertir un conjunto heterogéneo de piezas, cada una con sus propias inclinaciones, en una red ensamblada.

El proceso de traducción lo realizan los actores, humanos y no-humanos, que asumen roles de intermediarios y mediadores (Callon, 1986). Latour (2008) argumenta que los intermediarios son los que transportan significado o fuerza sin transformación, es decir son una representación o reflejo, lo que implica que al definir sus datos de entrada basta para definir sus datos de salida. Mientras que los mediadores pueden funcionar como uno, nada, varios o infinitos, por lo tanto, sus datos de entrada nunca predicen su salida ya que, su especificidad debe tomarse en cuenta cada vez, es decir, "los mediadores transforman, traducen, distorsionan y modifican el significado o los elementos que se supone que deben transportar" (Latour, 2008, p. 63).

Finalmente, Latour (2015) plantea que la utilidad de este análisis es recordar que lo que ordinariamente es denominado como incuestionable, solo es el resultado final de una serie de interacciones de oposición y apoyo entre actores. Por lo tanto, el seguimiento a la trayectoria de un proyecto desde esta perspectiva, puede ayudar a identificar el proceso de institucionalización o desinstitucionalización de una tecnología dentro de una organización, vinculando el cambio organizacional, tecnológico e institucional.

\section{Propuesta de vinculación teórica}

En consideración a lo anterior, se presenta una propuesta integrando los conceptos desarrollados desde la Teoría Actor-red -TAR- (Latour, 2008) y el Trabajo Institucional, para interrelacionar el cambio organizacional, el cambio tecnológico y el cambio institucional a partir de la introducción de una agencia distribuida entre los actores, humanos y no-humanos, que se reflejan en sus acciones o formas de trabajo y su afectación a los mecanismos institucionales. La propuesta plantea tres proposiciones que son enunciadas a continuación.

Proposición 1: En la implementación de una nueva tecnología se genera un proceso de traducción, al producirse conflictos con los actores, humanos y no-humanos, y los arreglos institucionales actuales, debido a las acciones emprendidas para cambiar o mantener los arreglos sociales y prácticas institucionalizadas en la organización.

La implementación de una nueva tecnología implica la llegada de nuevos actores al sistema o red de actores, reensamblando los arreglos sociales establecido entre los actores actuales (Latour, 2008). Esta implementación genera diversas interacciones y acciones entre los actores, alterando la organización, las tecnologías y las prácticas institucionalizadas, que no se estabilizan hasta que la nueva tecnología se ensambla con otros actores del sistema logrando su institucionalización. En este sentido, Latour (2015) argumenta que una vez introducida una tecnología, requiere de la construcción de un sistema que utiliza para mantener su posición tan irreversible como sea posible.

Entre más complejos y diversos son los cambios generados para implementar la nueva tecnología, se producen más contradicciones o conflictos con los actores del sistema y los arreglos institucionales actuales, por las acciones conjuntas entre actores humanos y no-humanos. Estas acciones se denominan formas de trabajo institucional (Lawrence \& Suddaby, 2008), por un lado, pueden mantener los arreglos sociales y 
prácticas institucionalizadas y, por otro, pueden alterar los mecanismos institucionales (Maguire \& Hardy, 2009; Scott, 2001), que buscan legitimar y, en consecuencia, institucionalizar la utilización de la nueva tecnología.

Para comprender la alteración del sistema o red de actores a partir de la implementación de una nueva tecnología, se propone un análisis del proceso de traducción (Callon \& Latour, 1981; Latour, 1986; Lawrence, 2017), y de esta forma entender cómo los actores, humanos y no-humanos, en las diferentes fases de la implementación, se movilizan para apoyar u oponerse a la inclusión de un nuevo actor no-humano al sistema, reensamblando el sistema actual. En este sentido, Yang y Wang (2013) proponen un análisis del proceso de traducción en cuatro pasos. Primero, la problematización, donde se convence a otros actores que existe un problema común, y se define la tecnología que puede ofrecer una solución. Segundo, la alienación, donde se incluyen los intereses subyacentes de cada actor, al demostrar las habilidades, el conocimiento u otros recursos necesarios que pueden permitir a los actores aceptar la solución como un medio para lograr sus objetivos, lo que motiva a los nuevos miembros a unirse a la red de actores. Tercero, el enrolamiento de los nuevos actores, en la red de actores para cumplir con sus roles y prácticas. Finalmente, la movilización de los actores para combinan sus recursos y en conjunto alcanzar la innovación (Yang \& Wang, 2013).

Proposición 2: La estabilidad de los arreglos sociales y prácticas institucionales, se soporta en una serie de mecanismos institucionales, que incluyen elementos regulatorios, elementos normativos, elementos cognitivos-culturales y elementos materiales.

En tanto construcciones sociales, las instituciones requieren que sus actores interactúen de forma permanente para mantener la estabilidad institucional (Berger \& Luckman, 1967), lo cual se realiza a través del apoyo, corrección y recreación de los mecanismos institucionales que garanticen su cumplimiento social (Lawrence \& Suddaby, 2006). En este sentido, los mecanismos institucionales vinculan las acciones y prácticas de los actores, es decir las formas de trabajo institucional, con elementos regulatorios, normativos y cognitivoculturales, que buscan dar estabilidad a los arreglos sociales y prácticas institucionales actuales.

Esta categorización de mecanismos institucionales deja de lado la tecnología y sus efectos sobre los arreglos sociales y las prácticas institucionales. Esto, a pesar que desde los estudios institucionales se ha discutido que los cambios tecnológicos generan cambios institucionales (Barley 1986; Battilana \& D’Aunno 2009; Clemens \& Cook, 1999). Recientemente se ha empezado a reconocer que se ha subestimado el rol de la tecnología como objeto material en relación con las instituciones (Dover \& Lawrence, 2010; Jones \& Massa, 2013; Monteiro \& Nicolini, 2014; Yang \& Wang, 2013). Dover y Lawrence (2010) argumentan que la tecnología y las instituciones tienen una dinámica compleja, que requiere de las interrelaciones entre las prácticas de individuos y organizaciones para mantener o cambiar los arreglos institucionales. Por su parte, Montero y Nicolini (2014) resaltan la necesidad de concebir la agencia como emergente y distribuida, argumentando que la preservación y cambio de los arreglos sociales sólo puede ser explicado cuando se reconoce la participación de las entidades materiales en los procesos de configuración institucional. Jones y Massa (2013) argumentan que las prácticas novedosas, que incluyen, entre otros, elementos materiales (Jones \& Massa, 2013), no reproducen las acciones previamente legitimadas o dadas por sentado, ya que proveen a los actores con un modelo alternativo viable que socava el orden institucional existente. Finalmente, Yang y Wang (2013) vinculan como nuevo mecanismo institucional a la tecnología, para comprender su rol en la transformación social. Pinch (2008) argumenta: "Las instituciones tienen una dimensión material ineludible... parte de la agencia que la materialidad trae a las instituciones es el trabajo de producir y reproducir (y algunas veces cambiar) la dimensión social de las instituciones" (Pinch, 2008, p. 466).

Por consiguiente, se propone la inclusión de una categoría de mecanismos materiales dentro de los mecanismos institucionales, para dar cuenta del rol de los actores no-humanos en la estabilidad o disrupción institucional. Estos mecanismos materiales se definen como los artefactos, software y sistemas de información, es decir, actores no-humanos que, a partir de su interrelación con los actores humanos, crean nuevas relaciones y ayudan a producir y reproducir los arreglos sociales. 
Proposición 3: La implementación de una nueva tecnología genera cambios continuos o discontinuos, en organizaciones, tecnologías e instituciones de forma interdependiente, cuando los actores humanos y no-humanos utilizan diferentes formas de trabajo institucional alterando alguno o todos los mecanismos institucionales que soportan las prácticas institucionales de la organización.

La relación entre cambio organizacional, tecnológico e institucional ha sido estudiada desde diferentes disciplinas y teorías demostrando su importancia, pero al mismo tiempo, generando una serie de vacíos en su entendimiento al centrarse en solo una dirección del cambio, por ejemplo, desde la teoría de la contingencia se caracteriza a la tecnología como determinante de los cambios organizacionales (Zammuto et al., 2007), mientras que desde la teoría socio-técnica se argumenta que son los sistemas sociales los que condicionan el uso de las tecnologías (Leonardi \& Barley, 2010). Por otro lado, desde la teoría institucional, algunos autores como Oliver (1991) y Pache y Santos (2010) argumentan que las organizaciones responden y se ajustan a partir de cambios en las demandas institucionales, mientras que Lawrence y Suddaby (2006) plantean que las organizaciones, a través de sus acciones, pueden alterar las prácticas institucionalizadas. A partir de este vacío se identifica la necesidad de estudiar el cambio tecnológico, organizacional e institucional como un proceso interdependiente, para lo que se requiere del entendimiento de las acciones conjuntas de actores humanos y no-humanos, reflejadas en la utilización de diferentes formas de trabajo institucional, en busca de la creación, modificación y disrupción de los arreglos sociales y prácticas institucionalizadas (Lawrence \& Suddaby, 2008), a partir de la alteración de alguno de los mecanismos institucionales: regulatorio, normativo, cognitivo o material.

Los actores no solo realizan acciones con el objetivo de cambiar organizaciones, tecnologías e instituciones, también las utilizan para mantener las actuales, desafiando u oponiéndose a las acciones de los actores que buscan la introducción de la nueva tecnología y el reensamblaje de los arreglos sociales actuales (Latour, 2015). En este sentido, las acciones que realizan los actores humanos con la mediación de actores nohumanos, reflejadas en la utilización de diferentes formas de trabajo institucional pueden mantener o alterar de incremental los mecanismos regulatorios a partir de reglas y políticas, los mecanismos normativos generando conformidad con los valores y normas actuales (Maguire \& Hardy, 2009), los mecanismos cognitivos con reglas culturales y significados compartidos (Hoffman, 1999), y los mecanismos materiales con la disponibilidad tecnológica y la interrelación de los actores no-humanos con los actores humanos, generan estabilidad en los actores actuales del sistema, sus relaciones, sus roles y de esta forma ayudan a producir y reproducir los arreglos sociales existentes y prácticas institucionalizadas, evitando la variación organizacional, tecnológica e institucional.

Por otro lado, entre mayor sea la alteración de los mecanismos institucionales para implementar una nueva tecnología, mayor será la modificación o disrupción en los arreglos sociales existentes y las prácticas institucionalizadas (Lawrence \& Suddaby, 2006), con nuevos actores, reglas, normas, valores, esquemas y marcos cognitivos, que generan nuevas prácticas, relaciones, roles y significados. Estos cambios, más que ser continuos o incrementales, es decir, congruentes con los esquemas existentes que se manejan dentro del sector como son el conocimiento, prácticas, significados y entendimientos compartidos (Rindova \& Petkova, 2007), son cambios discontinuos o disruptivos, es decir, incongruentes con los esquemas actuales y en las relaciones entre ellos (Christensen et al., 2009; Rindova \& Petkova, 2007).

En la Figura 6 se presentan gráficamente las tres proposiciones teóricas resultado de la revisión. Las proposiciones 1 y 2 muestran los conceptos que se relacionan cuando se introduce una nueva tecnología; la proposición 3 refleja los cambios que se generan por los esfuerzos de los actores para implementar una nueva tecnología. 
Proposición 3.

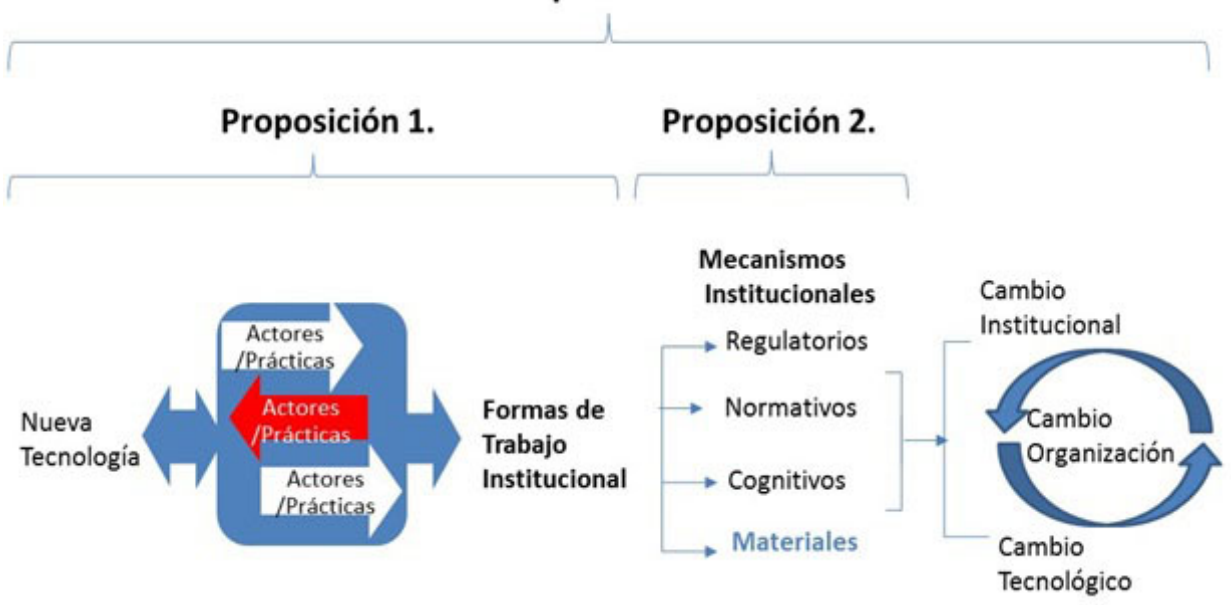

FIGURA 6

Proposiciones teóricas

Fuente: elaboración propia.

\section{CONCLUSIONES Y RECOMENDACIONES PARA FUTURAS INVESTIGACIONES}

En esta investigación presentamos una propuesta teórica que busca interrelacionar el cambio organización, tecnológico e institucional a partir de la integración de los conceptos de actor humano, actor no-humano, agencia distribuida, mecanismos institucionales y trabajo institucional, de la Teoría Actor-red y del Trabajo Institucional, para analizar las acciones conjuntas de los actores humanos y no-humanos en la creación, mantenimiento o disrupción de las prácticas institucionalizadas en una organización al implementarse una nueva tecnología. Varias conclusiones se desprenden de este trabajo.

Se evidencia la importancia de la interrelación entre organizaciones, tecnologías e instituciones, que ha sido estudiada desde diferentes disciplinas como la economía y la sociología, pero al mismo tiempo se identifican que estas diferentes aproximaciones generan un entendimiento parcial del cambio organizacional, tecnológico e institucional, el nivel, agencia y modo de cambio.

Primero se propone la integración de los conceptos de actor no-humano y traducción de Latour (2008) en el análisis de cambio organizacional, tecnológico e institucional, para entender como a partir de la agencia distribuida entre actores humanos y no-humano, se implementa una nueva tecnología en una organización, generando una serie de acciones que se oponen y apoyan esta implementación. Solucionando la problemática que se presenta, por un lado, en las teorías de ciclo de vida, evolución, contingencia y neo-institucional, que plantean una concepción pasiva de los actores organizacionales frente a elementos externos como la tecnología y la estructura. Por otro lado, en las teorías teleológica, dialéctica, constructivista, y de trabajo institucional donde se asigna a la agencia humana el rol central en el cambio.

Segundo, se propone la integración de las formas de trabajo institucional (Lawrence \& Suddaby, 2006), que busca analizar las acciones que de forma interrelacionada realizan los actores, humano y no-humanos, para crear, mantener, modificar, o generar disrupción de los arreglos sociales y prácticas institucionalizadas. Se espera que al utilizar las formas de trabajo institucional se puedan vincular los elementos micro de la organización (acciones y prácticas de los actores, humanos y no humanos, y arreglos sociales de la organización) con los elementos macro de las instituciones (arreglos sociales del sector como la regulación y prácticas institucionalizadas en el sector), que se ha identificado como una de las limitaciones en este tipo de estudios (Leonardi \& Barley, 2010). 
Finalmente, se plantea la vinculación de las formas de trabajo institucional utilizadas por los actores con los mecanismos institucionales, que se clasifican en regulatorios, normativos y cognitivo-culturales, para identificar como estas acciones y prácticas afectan los elementos que soportan las instituciones establecidas, analizando como la alteración de alguno de estos mecanismos generan cambios organizacionales, tecnológicos e institucionales de manera interdependiente. Además, se propone la inclusión de una categoría de mecanismos materiales dentro de los mecanismos institucionales, para dar cuenta de su rol en la estabilidad o disrupción institucional.

Este estudio tiene implicaciones para futuras investigaciones. En primer lugar, a partir de esta revisión teórica y propuesta de vinculación teórica se podría generar un esquema metodológico para facilitar el estudio de esta interrelación, integrando las herramientas analíticas propuestas por la Teoría Actor-Red, para mapear los actores y acciones, tanto de humanos como no-humanos, que se interrelacionan con la implementación de nuevas tecnologías, y para realizar un análisis del proceso de traducción de la institucionalización de nuevas tecnologías. Estas propuestas se presentan como proposiciones teóricas que buscan guiar futuras investigaciones a responder preguntas como ¿cuál es el rol de las tecnologías en permitir o restringir la innovación dentro de las organizaciones?, ¿cómo las prácticas institucionales existentes pueden impedir la innovación? y, ¿cómo pueden los opositores a la innovación utilizar las prácticas institucionales para resistirse a los cambios?

Finalmente, este tipo de investigaciones podrían generar insumos que pueden ser utilizados a nivel de las organizaciones en el diseño de programas para la institucionalización de las nuevas prácticas relacionadas con el proceso de implementación de nuevas tecnologías.

En conclusión, esta investigación busca contribuir al entendimiento de cómo y por qué los actores, tanto humanos como no-humanos, reensamblan y reconfiguran las prácticas institucionalizadas y cómo esas acciones llevan a la implementación o no de nuevas tecnologías y a adaptaciones, mutaciones y otros cambios tecnológicos y organizacionales.

\section{Agradecimientos}

Los autores agradecemos a la Pontificia Universidad Javeriana Cali, por el apoyo en el desarrollo de esta investigación a través del Programa de Formación Doctoral de Profesores, a la Universidad Nacional de Colombia, específicamente a la Escuela de Diseño Industrial de la Facultad de Artes por facilitar el Convenio (230614) con la Universidad del Valle, y al grupo de investigación ILLANTIO de la Escuela de Diseño Industrial de la Universidad Nacional de Colombia, por brindar los espacios de reflexión y trabajo para realizar esta investigación.

\section{REFERENCIAS}

Barad, K. (2003). Posthumanist performativity: toward an understanding of how matter comes to matter. Signs, 28(3), 801-831. http://dx.doi.org/10.1086/345321

Barad, K. (2007). Meeting the University Halfway: Quantum physics and the entanglement of matter and meaning. Durham: Duke University Press.

Barley, S. (1986). Technology as an occasion for structuring: Evidence from observations of CT scanners and the social order of radiology departments. Administrative Science Quarterly, 31, 78-108. http://dx.doi.org/10.2307/239 2767

Battilana, J., \& D'Aunno, T. (2009). Institutional work and the paradox of embedded agency. In T. Lawrence, R. Suddaby, y B. Leca (eds.), Institutional work: Actors and agency in institutional studies of organization (pp. 31-58). Cambridge: Cambridge University Press. http://dx.doi.org/10.1017/CBO9780511596605.001 
Berger, P., \& Luckman, T. (1967). The social construction of reality: a treatise in the sociology of knowledge. New York: Anchor Books. http://dx.doi.org/10.1093/bjsw/bcn068.

Beunza, D., \& Ferraro, F. (2019). Performative work: Bridging performativity and institutional theory in the responsible investment field. Organization Studies, 40(4), 515-543. https://doi.org/10.1177/0170840617747 917

Bijker, W. (1995). Of Bicycles, Bakekites, and Bulbs: Toward a Theory of Sociotechnical Change. Cambridge: MIT Press. http://sciencepolicy.colorado.edu/students/envs_5110/bijker.pdf

Callon, M. (1986). Some elements of a sociology of translation: Domestication of scallops and the fishermen of St Brieuc Bay. In J. Law (ed.), Power, Action and Belief: A New Sociology of Knowledge? (pp. 196-233). London: Routledge.

Clemens, E., \& Cook, J. (1999). Politics and institutionalism: Explaining durability and change. Annual Review of Sociology, 25, 441-466. http://dx.doi.org/10.1146/annurev.soc.25.1.441

Dancin, M., Goodstein, J., \& Scott, R. (2002). Institutional Theory and Institutional Change: introduction to the special research Forum. Academy of Management Journal, 45(1), 43-56. http://dx.doi.org/10.2307/3069284

DiMaggio, P. (1988). Interest and agency in institutional theory. En L. Zucker (Ed.) Institutional patterns and organizations: culture and environment (pp. 3-32). Cambridge, MA: Ballinger.

DiMaggio, P., \& Powell, W. (1983). The iron cage revisited: institutional isomorphism and collective rationality in organizational fields. American Sociological Review, 48(2), 147-160. https://www.jstor.org/stable/2095101

Dosi, G. (1982). Technological paradigms and technological trajectories. Research Policy, 11, 147-162. https://doi.o $\mathrm{rg} / 10.1016 / 0048-7333(82) 90016-6$

Douglas, M. (1986). How Institutions think. London: Routledge.

Dover, G., \& Lawrence, T. (2010). Technology, institutions and entropy: Understanding the critical and creative role of maintenance work. In Technology and organization: Essays in honour of Joan Woodward (v. 29, pp. 259-264). Bingley, UK: Emerald Group. http://dx.doi.org/10.1108/S0733-558X(2010)0000029019.

Gawer, A. \& Phillips, N. (2013). Institutional work as logics shift: The case of intel's transformation to platform leader. Organization Studies, 34(8), 1035-1071. http://dx.doi.org/10.1177/0170840613492071

Greenwood, R., \& Suddaby, R. (2006). Institutional entrepreneurship in mature fields: The five accounting firms. Academy of Management Journal, 49, 27-48. http://dx.doi.org/10.5465/AMJ.2006.20785498

Greenwood, R., Suddaby, R., \& Hinings, C. (2002). Theorizing change: the role of professional associations in the transformation of institutionalized fields. Academy of Management Journal, 45(1), 58-80. http://dx.doi.org/10 $.2307 / 3069285$

Hoffman, A. (1999). Institutional evolution and change: environmentalism and the U.S. Chemical Industry. Academy of Management Journal, 42(4), 351-371. http://dx.doi.org/10.2307/257008

Iacono, S., \& Kling, R. (2001). Computerization movements: The rise of Internet and distant forms of work. In J. Yates \& J. Van Maanen (eds.), Information technology and organizational transformation: History, rhetoric, and practice (pp. 93-136). California: Sage Publications.

Jones, C., \& Massa, F. (2013). From novel practice to consecrated exemplar: unity temple as a case of institutional evangelizing. Organization Studies, 34(8), 1099-1136. http://dx.doi.org/10.1177/0170840613492073

Kling, R. (1991). Computerization and Social Transformation. Science, Technology \& Human Values, 16(3), 342-367. http://dx.doi.org/10.1177/016224399101600304

Kling, R., \& Iacono, S. (1988). The mobilization of support for computerization: The role of computerization movements. Social Problems, 35(3), 226-243. http://dx.doi.org/10.2307/800620

Knorr-Cetina, K. (1997). Sociality with objects: Social relations in postsocial knowledge societies. Theory, Culture \& Society, 14(4), 1-30. https://doi.org/10.1177/026327697014004001

Latham, R. \& Sassen, S. (2005). Digital formations: IT and new architectures in the global realm. Princeton: Princeton University Press. 
Latour, B. (1987). Science in action: how to follow scientist and engineers through society. Cambridge, MA: Harvard University Press.

Latour, B. (1992). Where are the missing masses? The sociology of a few mundane artifacts. In W. Bijker \& J. Law (eds.), Shaping technology/building society: Studies in sociotechnical change (pp. 225-258). Cambridge, Mass: MIT Press.

Latour, B. (2008). Reensamblar lo social: una introducción a la teoría del actor-red. Buenos Aires: Manantial.

Latour, B. (2015). Scientific Humanities. Material curso en Coursera (junio - julio 2015), desde http://www.course ra.org

Law, J. (1992). A sociology of monsters: Essays on power, technology and domination. London: Routlege Sociological Review Monograph.

Law, J. (1987). Technology and heterogeneous engineering: The case of Portuguese expansion. In W. Bijker, T. Hughes \& T. Pinch (eds.), The Social Construction of Technological: New Directions in the Sociology and History of Technology Studies (pp. 111-134). Cambridge, MA: The MIT Press.

Lawrence, T., Leca, B. \& Zilber, T. (2013). Institutional work: current research, new directions and overlooked issues. Organization Studies, 34(8), 1023-1033. http://dx.doi.org/10.1177/0170840613495305

Lawrence, T. \& Suddaby, R. (2006). Institutions and Institutional Work. In S. Clegg, C. Hardy, T. Lawrence, \& W. Nord, (eds.), Handbook of organization studies (pp. 215-254), 2nd ed. Londres: Sage Publications.

Lawrence, T., Suddaby, R. \& Leca, B. (2009). Institutional Work: actors and agency in institutional studies of organizations. Cambridge: Cambridge University Press.

Lawrence, T., Suddaby, R. \& Leca, B. (2011). Institutional Work: refocusing institutional studies of organizations. Journal of Management Inquiry, 20(1), 52-58. http://dx.doi.org/10.1177/105649261038722

Leonardi, P., \& Barley, S. (2008). Materiality and change: challenges to build better theory about technology and organizing. Information and Organization, 18, 159-176. http://dx.doi.org/10.1016/j.infoandorg.2008.03.001

Leonardi, P., \& Barley, S. (2010). What's under construction here? Social action, materiality, and power in constructivist studies of technology and organizing. Academy of Management Annals, 4(1), 1-51. http:// dx.doi.org/10.1080/19416521003654160

Maguire, S., \& Hardy, C. (2009). Discourse and deinstitutionalization: the decline of DDT. Academy of Management Journal, 52(1), 148-178. http://dx.doi.org/10.5465/AMJ.2009.36461993

Meyer, J., \& Rowan, B. (1977). Institutionalized organizations: Formal structure as myth and ceremony. American Journal of Sociology, 83(2), 340-363. https://doi.org/10.1086/226550

Montero, P., \& Nicolini, D. (2014). Recovering materiality in institutional work: prizes as an assemblage of human and material entities. Journal of Management Inquiry, 1-21. http://dx.doi.org/10.1177/1056492614546221

Nelson, R., \& Winter, S. (1982). An Evolutionary Theory of Economic Change. Cambridge, Mass: Harvard University Press.

Oliver, C. (1991). Strategic Responses to Institutional Processes. Academy of Management Review, 16(1), 145-179. h ttp://dx.doi.org/10.5465/AMR.1991.4279002

Oliver, C. (1992). The antecedents of deinstitututionalization. Organization Studies, 13(4), 563-588. http://dx.doi.o $\mathrm{rg} / 10.1177 / 017084069201300403$

Orlikowski, W. (2010). The sociomateriality of organizational life: considering technology in management research. Cambridge Journal of Economics, 34, 125-141. http://dx.doi.org/10.1093/cje/bep058

Orlikowski, W., \& Scott, S. (2008). Sociomateriality: challenging the separation of technology, work and organization. Academy of Management Annals, 2(1), 433-474. http://dx.doi.org/10.1080/19416520802211644

Pache, A., \& Santos, F. (2010). When Worlds Collide: The internal dynamics of organizational responses to conflicting institutional demands. Academy of Management Review, 35(3), 455-476. https://doi.org/10.5465/amr.35.3.zo $\mathrm{k} 455$

Pickering, A. (1995). The mangle of practice: time, agency and science. Chicago: University of Chicago Press.

Pinch, T. (2008). Technology and institutions: living in a material world. Theory and Sociology, 37, 461-483. http:// dx.doi.org/10.1007/s11186-008-9069-x 
Pinch, T., \& Bijker, W. (1984). The social construction of facts and artefacts: or how the sociology of science and the sociology of technology might benefit each Other. Social Studies of Science, 14(3), 399-441. http://dx.doi.org/ $10.1177 / 030631284014003004$

Powell, W., \& DiMaggio, P. (1991). The new Institutionalism in Organizational Analysis. Chicago: University of Chicago Press

Powell, W., \& Grodal, S. (2006) Networks of innovators (cap. 3). In J. Fagerberg, D. Mowery, \& R. Nelson. The Oxford Handbook of Innovation. Oxford: Oxford University Press.

Raviola, E., \& Norback, M. (2013). Bringing technology and meaning into institutional work: Making news at an Italian business newspaper. Organization Studies, 34(8), 1171-1194. http://dx.doi.org/10.1177/01708406134 92077

Rindova, V., \& Petkova, A. (2007). When is a new thing a good thing? Technological change, product form design, and perceptions of value for product innovations. Organization Science, 18(2), 217-232. http://dx.doi.org/10. $1287 /$ orsc. 1060.0233

Sayes, E. (2014). Actor-Network Theory and methodology: just what does it mean to say that nonhumans have agency? Social Studies of Science, 44(1), 134-149. http://dx.doi.org/10.1177/0306312713511867

Scott, R. (2001). Institutions and Organizations, Second Edition. Londres: Sage Publications.

Scott, R., Ruef, M., Mendel, P., \& Caronna, C. (2000). Institutional change and healthcare organizations: from professional dominance to managed care. Chicago: The University of Chicago Press.

Selznick, P. (1957). Leadership in administration: a sociological interpretation. Berkeley: University of California Press.

Seo, M., \& Creed, D. (2002). Institutional Contradictions, Praxis, and Institutional Change: a dialectical perspective. Academy of Management Review, 27(2), 232-247. http://dx.doi.org/10.5465/AMR.2002.6588004

Suchman, L. (2007). Human-machine reconfigurations: Plans and situated actions. Cambridge: Cambridge University Press.

Thornton, P., Ocasio, W., \& Lounsbury, M. (2012). The institutional logics perspective: a new approach to culture, structure, and process. Oxford: Oxford University Press.

Tryggestad, K., \& Georg, S. (2011). How objects shape logics in construction. Culture and Organization, 17(3), 181-197. https://doi.org/10.1080/14759551.2011.569383

Van de Ven, A. (2007). Engaged Scholarship: A guidefor organizational and social research. Oxford: Oxford University Press.

Van de Ven, A., \& Poole, M. (1995). Explaining development and change in organizations. Academy of Management Review, 20(3), 510-540. http://dx.doi.org/10.5465/AMR.1995.9508080329

Waldorff, S. (2013). Accounting for organizational innovations: Mobilizing institutional logics in translation. Scandinavian Journal of Management, 29(3), 219-234. https://doi.org/10.1016/j.scaman.2013.03.010

Williams, R., \& Edge, D. (1996). The social shaping of technology. Research policy, 25, 865-899. http://dx.doi.org/1 $0.1016 / 0048-7333(96) 00885-2$

Yang, G., \& Wang, R. (2013). The Institutionalization of an Electronic Marketplace in China, 1998-2010. Journal of Product Innovation Management, 30(1), 96-109. https://doi.org/10.1111/j.1540-5885.2012.00989.x

Zammuto, R., Griffith, T., Majchrzak, A., Dougherty, D., \& Faraj, S. (2007). Information technology and the changing fabric of organization. Organization Science, 18(5), 749-762. http://dx.doi.org/10.1287/orsc.1070.0307

\section{Notas}

* Artículo de revisión.

1 Campo organizacional se refiere a "aquellas organizaciones que, en agregado, constituyen un área reconocida de la vida institucional: proveedores claves, consumidores de recursos y productos, agencias regulatorias y otras organizaciones que producen productos o servicios similares" (DiMaggio \& Powell, 1983, p. 148). 


\section{Licencia Creative Commons CC BY 4.0}

Sugerencia de citación: Gutiérrez R., V. A., Aguilar Z., J. J., y Medina V., J. E. (2019). Cambio organizacional, institucional y tecnológico: una aproximación desde la teoría actor-red y el trabajo institucional. Cuadernos de Administración, 32(59). DOI: https://doi.org/10.11144/Javeriana.cao32-59.coit 